DISTA-UPO/05

LMU-ASC 66/05

MPP-2005-119

\title{
Noncommutative Geometry and Gravity
}

\author{
Paolo Aschieri ${ }^{1}$, Marija Dimitrijević ${ }^{2,3,4}$, Frank Meyer ${ }^{2,3, \dagger}$ and Julius Wess ${ }^{2,3}$ \\ ${ }^{1}$ Dipartimento di Scienze e Tecnologie Avanzate \\ Universitá del Piemonte Orientale, and INFN \\ Via Bellini 25/G 15100 Alessandria, Italy \\ ${ }^{2}$ Arnold Sommerfeld Center for Theoretical Physics \\ Universität München, Fakultät für Physik \\ Theresienstr. 37, 80333 München, Germany \\ ${ }^{3}$ Max-Planck-Institut für Physik \\ Föhringer Ring 6, 80805 München, Germany \\ ${ }^{4}$ University of Belgrade, Faculty of Physics \\ Studentski trg 12, 11000 Beograd, Serbia and Montenegro
}

\begin{abstract}
We study a deformation of infinitesimal diffeomorphisms of a smooth manifold. The deformation is based on a general twist. This leads to a differential geometry on a noncommutative algebra of functions whose product is a star-product. The class of noncommutative spaces studied is very rich. Non-anticommutative superspaces are also briefly considered.

The differential geometry developed is covariant under deformed diffeomorphisms and it is coordinate independent. The main target of this work is the construction of Einstein's equations for gravity on noncommutative manifolds.
\end{abstract}

PACS: 02.40.Gh, 02.20.Uw, 04.20.-q, 11.10.Nx, 04.60.-m. 2000 MSC: 83C65, 53D55, 81R60, 58B32 E-mail: aschieri,dmarija,meyerf,wess@theorie.physik.uni-muenchen.de 


\section{Contents}

1 Introduction $\quad 1$

2 Deformation by twists $\quad 4$

2.1 Hopf algebras from Lie algebras . . . . . . . . . . . . . . . . . 4

2.2 The twist . . . . . . . . . . . . . . . . . . . . . . . . . . . . . . . . . .

2.3 Examples of twists . . . . . . . . . . . . . . . . . 8

2.3.1 Deformed Superspace . . . . . . . . . . . . . . . 10

2.4 The deformed Hopf algebra $U \Xi^{\mathcal{F}} \ldots \ldots \ldots$. . . . . . . . . . . . . 11

3 Representations $\quad 13$

3.1 Module Algebras . . . . . . . . . . . . . . . . . . . . . . . 13

3.2 Examples of Module algebras . . . . . . . . . . . . . . . . . . . . . . . . . . . . . . . . . . 15

$3.3 U \Xi_{\star}$ is a Hopf algebra . . . . . . . . . . . . . . . . . . . 15

4 Representations of deformed infinitesimal diffeomorphisms $\quad 17$

4.1 Tensorfields .......................... 18

4.1.1 Vectorfields $\Xi_{\star}$ are an $A_{\star}$-bimodule . . . . . . . . . . . . . . 19

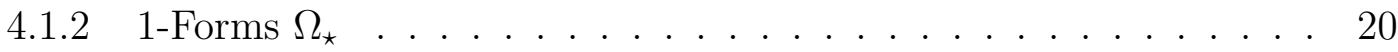

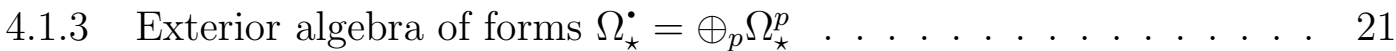

$4.2 \star$-Pairing between 1 -forms and vectorfields . . . . . . . . . . . 21

5 Covariant Derivative 22

6 Torsion and Curvature $\quad 24$

7 Metric and Einstein Equations $\quad 28$

8 Conjugation $\quad 30$

$\begin{array}{ll}\text { A Appendix } & 32\end{array}$

A.1 Proof that $U \Xi^{\mathcal{F}}$ is a Hopf Algebra . . . . . . . . . . . . . . . . . . . . 32

A.2 $\star$-Jacoby identity . . . . . . . . . . . . . . . . . 34

A.3 Associativity of the $\star$-product on superspace . . . . . . . . . . . . 35

\section{Introduction}

The study of the structure of spacetime at Planck scale, where quantum gravity effects are non-negligible, is one of the main open challenges in fundamental physics. Since the dynamical variable in Einstein general relativity is spacetime itself (with its metric 
structure), and since in quantum mechanics and in quantum field theory the classical dynamical variables become noncommutative, one is strongly lead to conclude that noncommutative spacetime is a feature of Planck scale physics. This expectation is further supported by Gedanken experiments that aim at probing spacetime structure at very small distances. They show that due to gravitational backreaction one cannot test spacetime at Planck scale ${ }^{1}$. Its description as a (smooth) manifold becomes therefore a mathematical assumption no more justified by physics. It is then natural to relax this assumption and conceive a more general noncommutative spacetime, where uncertainty relations and discretization naturally arise. In this way one can argue for the impossibility of an operational definition of continuous Planck lenght spacetime (i.e., a definition given by describing the operations to be performed for at least measuring spacetime by a Gedanken experiment). A dynamical feature of spacetime could be incorporated at a deeper kinematical level. As an example compare Galilean relativity to special relativity. Contraction of distances and time dilatation can be explained in Galilean relativity: they are a consequence of the interaction between ether and the body in motion. In special relativity they have become a kinematical feature.

This line of thought has been pursued in previous works, starting with [1], [2], and more recently in [3] - [15] .

Notice that uncertainty relations in position measurements are also in agreement with string theory models [16]. Moreover, non-perturbative attempts to describe string theories have shown that a noncommutative structure of spacetime emerges [17].

A first question to be asked in the context we have outlined is whether one can consistently deform Riemannian geometry into a noncommutative Riemannian geometry. We address this question by considering deformations of the algebra of functions on a manifold obtained via a quite wide class of $\star$-products. In this framework we successfully construct a noncommutative version of differential and of Riemannian geometry, and we obtain the noncommutative version of Einstein equations.

Even without physical motivations, the mathematical structure of deformed spaces is a challenging and fruitful research arena. It is very surprising how well $\star$-noncommutative structures can be incorporated in the framework of differential geometry.

The $\star$-products we consider are associated with a deformation by a twist $\mathcal{F}$ of the Lie algebra of infinitesimal diffeomorphisms on a smooth manifold $M$. Since $\mathcal{F}$ is an arbitrary twist, we can consider it as the dynamical variable that determines the possible

\footnotetext{
${ }^{1}$ For example, in relativistic quantum mechanics the position of a particle can be detected with a precision at most of the order of its Compton wave length $\lambda_{C}=\lambda / m c$. Probing spacetime at infinitesimal distances implies an extremely heavy particle that in turn curves spacetime itself. When $\lambda_{C}$ is of the order of the Planck length, the spacetime curvature radius due to the particle has the same order of magnitude and the attempt to measure spacetime structure beyond Planck scale fails.
} 
noncommutative structures of spacetime.

In Section 2 we construct the universal enveloping algebra $U \Xi$ of the Lie algebra of vectorfields, and we give a pedagogical description of its Hopf algebra structure. The twists we consider are elements $\mathcal{F} \in U \Xi \otimes U \Xi$. The notion of twist of a Lie algebra is well known [18,19]. Multiparametric twists appear in [20]. Other examples of twists (Jordanian deformations) are in [22], [23] and [24]. In the context of deformed Poincaré group and Minkowski space geometry twists have been studied in [25], [26] (multiparametric deformations), and in [27], [28], [29] [30], [31] (Moyal-Weyl deformations), see also [32].

In the context of Connes noncommutative geometry, the noncommutative torus, the noncommutative spheres [33] and further noncommtative manifolds (so-called isospectral deformations) considered in [33], and in [34], [35], are noncommutative manifolds whose deformed algebra of functions is along the lines of Rieffel's twists [37]; see [38] and, for the four-sphere in [33], see [36], [39].

Our contribution in this section is to consider the notion of twist in the context of an infinite dimensional Lie algebra, that of vectorfields on $M$. Several examples of twists and of their corresponding $\star$-noncommutative algebra of funtcions are then presented. We also extend this notion to the case where $M$ is superspace, and describe in a sound mathematical setting a very general class of twists on superspace.

We conclude Section 2 by recalling the construction of the Hopf algebra $U \Xi^{\mathcal{F}}[19]$. This Hopf algebra is closely related to the Hopf algebra of deformed infinitesimal diffeomorhisms.

We begin Section 3 by recalling some known facts about Hopf algebra representations and then construct the algebra $U \Xi_{\star}$ (with product $\star$ ) as a module algebra on which $U \Xi^{\mathcal{F}}$ acts. The space of vectorfields has a deformed Lie bracket that is realized as a deformed commutator in $U \Xi_{\star}$. We have constructed the deformed Lie algebra of infinitesimal diffeomorphisms (infinitesimal $\star$-diffeomorphisms). We then construct a natural Hopf algebra structure on $U \Xi_{\star}$ which proves that vectorfields form a deformed Lie algebra in the sense of [40], see also [41], [42], and [43] p. 41. It can also be proven that $U \Xi_{\star}$ and $U \Xi^{\mathcal{F}}$ are isomorphic Hopf algebras [44]. In [14], [45] and [46] (where $\theta^{\mu \nu}$-constant noncommutativity is considered) the Hopf algebra $U \Xi^{\mathcal{F}}$ rather than $U \Xi_{\star}$ is used.

In Section 4 we study the $\star$-action of the Hopf algebra of infinitesimal $\star$-diffeomorphisms on the algebra of noncommutative functions $A_{\star} \equiv F u n_{\star}(M)$ and on $U \Xi_{\star}$. In the same way that $A \equiv F u n(M)$ and $U \Xi$ were deformed in Section 3, we here deform the algebra of tensorfields $\mathcal{T}$ into $\mathcal{T}_{\star}$ and then study the action of $\star$-diffeomorphisms on $\mathcal{T}_{\star}$. As a further example we similarly proceed with the algebra of exterior forms.

We then study the pairing between vectorfields and 1-forms, and its $A_{\star}$-linearity properties. Moving and dual comoving frames (vielbein) are introduced. As in the 
commutative case, (left) $A_{\star}$-linear maps $\Xi_{\star} \rightarrow A_{\star}$ are the same as 1 -forms. More in general tensorfields can be equivalently described as (left) $A_{\star}$-linear maps.

In Section 5 we define the $\star$-covariant derivative in a global coordinate independent way. Locally the covariant derivative is completely determined by its coefficients $\Gamma_{\mu \nu}^{\sigma}$. Using the deformed Leibniz rule for vectorfields we extend the covariant derivative to all type of tensorfields.

In Section 6 torsion, curvature and the Ricci tensors are defined as (left) $A_{\star}$-linear maps on vectorfields. The $A_{\star}$-linearity property is a strong requirement that resolves the ambiguities in the possible definitions of these noncommutative tensorfields.

In Section 7 we define the metric as an arbitrary $\star$-symmetric element in the $\star$ tensorproduct of 1 -forms $\Omega_{\star} \otimes_{\star} \Omega_{\star}$. Using the pairing between vectorfields and 1 -forms the metric is equivalently described as an $A_{\star}$-linear map on vectorfields, $(u, v) \mapsto \mathrm{g}(u, v)$. The scalar curvature is then defined and Einstein equations on $\star$-noncommutative space are obtained. Again the requirement of $A_{\star}$-linearity uniquely fixes the possible ambiguities arising in the noncommutative formulation of Einstein gravity theory.

In Section 8 we study reality conditions on noncommutative functions, vectorfields and tensorfields. If the twist $\mathcal{F}$ satifies a mild natural extra condition then all the geometric constructions achieved in the previous sections admit a real form.

\section{Deformation by twists}

\subsection{Hopf algebras from Lie algebras}

Let us first recall that the (infinite dimensional) linear space $\Xi$ of smooth vectorfields on a smooth manifold $M$ becomes a Lie algebra through the map

$$
\text { [ ] : } \begin{aligned}
\Xi \times \Xi & \rightarrow \Xi \\
(u, v) & \mapsto[u v] .
\end{aligned}
$$

The element $[u v]$ of $\Xi$ is defined by the usual Lie bracket

$$
[u v](h)=u(v(h))-v(u(h)) \text {. }
$$

We shall always denote vectorfields by the letters $u, v, z, \ldots$ and functions on $M$ by $f$, $g, h, \ldots$

The Lie algebra of vectorfields (i.e. the algebra of infinitesimal diffeomorphisms) can also be seen as an abstract Lie algebra without referring to the smooth manifold $M$ anymore. This abstract algebra can be extended to a Hopf algebra by first defining the universal enveloping algebra $U \Xi$ that is the tensor algebra (over $\mathbb{C}$ ) generated by the elements of $\Xi$ and the unit element 1 modulo the left and right ideal generated by all 
elements $u v-v u-[u v]$. The elements $u v$ and $v u$ are elements in the tensor algebra and $\left[\begin{array}{ll}u & v\end{array}\right]$ is an element of $\Xi$. We shall denote elements of the universal enveloping algebra $U \Xi$ by $\xi, \zeta, \eta, \ldots$

The algebra $U \Xi$ has a natural Hopf algebra structure $[47,48]$. On the generators $u \in \Xi$ and the unit element 1 we define

$$
\begin{array}{ll}
\Delta(u)=u \otimes 1+1 \otimes u, & \Delta(1)=1 \otimes 1, \\
\varepsilon(u)=0, & \varepsilon(1)=1, \\
S(u)=-u, & S(1)=1 .
\end{array}
$$

Here $\Delta$ is the coproduct (from which the Leibniz rule for vectorfields follows), $S$ is the antipode (or coinverse) and $\varepsilon$ the counit. The maps $\Delta, \varepsilon$ and $S$ satisfy the following relations

$$
\begin{aligned}
\Delta(u) \Delta(v)-\Delta(v) \Delta(u) & =[u v] \otimes 1+1 \otimes[u v]=\Delta([u v]), \\
\varepsilon(u) \varepsilon(v)-\varepsilon(v) \varepsilon(u) & =\varepsilon([u v]), \\
S(v) S(u)-S(u) S(v) & =v u-u v=S([u v]) .
\end{aligned}
$$

This allows us to extend $\Delta$ and $\varepsilon$ as algebra homomorphisms and $S$ as antialgebra homomorphism to the full enveloping algebra, $\Delta: U \Xi \rightarrow U \Xi \otimes U \Xi, \varepsilon: U \Xi \rightarrow \mathbb{C}$ and $S: U \Xi \rightarrow U \Xi$

$$
\begin{aligned}
\Delta(\xi \zeta) & :=\Delta(\xi) \Delta(\zeta) \\
\varepsilon(\xi \zeta) & :=\varepsilon(\xi) \varepsilon(\zeta), \\
S(\xi \zeta) & :=S(\zeta) S(\xi) .
\end{aligned}
$$

There are three more propositions that have to be satisfied for a Hopf algebra (we denote by $\mu$ the product in the algebra)

$$
\begin{aligned}
(\Delta \otimes i d) \Delta(\xi) & =(i d \otimes \Delta) \Delta(\xi) \\
(\varepsilon \otimes i d) \Delta(\xi) & =(i d \otimes \varepsilon) \Delta(\xi)=\xi \\
\mu(S \otimes i d) \Delta(\xi) & =\mu(i d \otimes S) \Delta(\xi)=\varepsilon(\xi) 1
\end{aligned}
$$

It is enough to prove (2.6) on the generators $u, 1$ of $U \Xi$. We prove the first of them for the coproduct defined in (2.3) using the Sweedler notation $\Delta(u)=u_{1} \otimes u_{2}$ (where a sum over $u_{1}$ and $u_{2}$ is understood), in this explicit case $\Delta(u)=u_{1} \otimes u_{2}=u \otimes 1+1 \otimes u$,

$$
\begin{aligned}
(\Delta \otimes i d) \Delta(u) & =\Delta\left(u_{1}\right) \otimes u_{2} \\
& =u_{1_{1}} \otimes u_{1_{2}} \otimes u_{2} \\
& =(u \otimes 1+1 \otimes u) \otimes 1+1 \otimes 1 \otimes u
\end{aligned}
$$


and

$$
\begin{aligned}
(i d \otimes \Delta) \Delta(u) & =u_{1} \otimes \Delta\left(u_{2}\right) \\
& =u_{1} \otimes u_{2_{1}} \otimes u_{2_{2}} \\
& =u \otimes 1 \otimes 1+1 \otimes(u \otimes 1+1 \otimes u)
\end{aligned}
$$

Comparing (2.7) and (2.8) we see that the first condition of (2.6) is satisfied.

After proving the remaining conditions of (2.6) on the generators of $U \Xi$ we have constructed the Hopf algebra $(U \Xi, \cdot \Delta, S, \varepsilon)$, where $\cdot$ denotes the multiplication map in $U \Xi$; sometimes we denote it by $\mu$ and frequently omit any of the symbols $\cdot$ and $\mu$. With abuse of notation we frequently write $U \Xi$ to denote the Hopf algebra $(U \Xi, \cdot, \Delta, S, \varepsilon)$. This Hopf algebra is cocommutative because $\Delta=\Delta^{o p}$ where $\Delta^{o p}=\sigma \circ \Delta$ with $\sigma$ the flip map $\sigma(\xi \otimes \zeta)=\zeta \otimes \xi$.

We will extend the notion of enveloping algebra to formal power series in $\lambda$, and we will correspondingly consider the Hopf algebra $(U \Xi[[\lambda]], \cdot, \Delta, S, \varepsilon)$. In the sequel for sake of brevity we will often denote $U \Xi[[\lambda]]$ by $U \Xi$.

\subsection{The twist}

Definition 1. A twist $\mathcal{F}$ is an element $\mathcal{F} \in U \Xi[[\lambda]] \otimes U \Xi[[\lambda]]$ that is invertible and that satisfies

$$
\begin{gathered}
\mathcal{F}_{12}(\Delta \otimes i d) \mathcal{F}=\mathcal{F}_{23}(i d \otimes \Delta) \mathcal{F}, \\
(\varepsilon \otimes i d) \mathcal{F}=1=(i d \otimes \varepsilon) \mathcal{F},
\end{gathered}
$$

where $\mathcal{F}_{12}=\mathcal{F} \otimes 1$ and $\mathcal{F}_{23}=1 \otimes \mathcal{F}$

In our context we in addition require ${ }^{2}$

$$
\mathcal{F}=1 \otimes 1+\mathcal{O}(\lambda)
$$

Property (2.9) states that $\mathcal{F}$ is a two cocycle, and it will turn out to be responsible for the associativity of the $\star$-products to be defined. Property (2.10) is just a normalization condition. From (2.11) it follows that $\mathcal{F}$ can be formally inverted as a power series in $\lambda$. It also shows that the geometry we are going to construct has the nature of a deformation, i.e. in the 0 -th order in $\lambda$ we recover the usual undeformed geometry.

Using the twist $\mathcal{F}$ we now proceed to deform the commutative geometry on $M$ into the twisted noncommutative one. The guiding principle is the observation that every time we have a linear map $X \otimes Y \rightarrow Z$, or a linear map $Z \rightarrow X \otimes Y$, where $X, Y, Z$

\footnotetext{
${ }^{2}$ Actually it is possible to show that $(2.11)$ is a consequence of $(2.9),(2.10)$ and of $\mathcal{F}$ being at each order in $\lambda$ a finite sum of finite products of vectorfields
} 
are vectorspaces, and where $U \Xi$ acts on $X, Y$ and $Z$, we can combine this map with an action of the twist. In this way we obtain a deformed version of the initial linear map. To preserve algebraic properties of the original maps very particular actions of the twist $\mathcal{F}$ have to be used.

As an example let $X=Y=Z=A$ where $A \equiv F u n(M) \equiv C^{\infty}(M)[[\lambda]]$ is the algebra of smooth functions on $M$. The elements of $U \Xi$ act on $A$ by the natural extension of the Lie derivative. The Lie derivative on $\operatorname{Fun}(M)$ associated with the vectorfield $v$ is defined as follows

$$
\mathcal{L}_{v}(h)=v(h) \in A=\operatorname{Fun}(M),
$$

where $v \in \Xi$ and $h \in F u n(M)$. From equation (2.12) follows that the map

$$
v \mapsto \mathcal{L}_{v}
$$

satisfies

$$
\mathcal{L}_{v^{\prime}} \mathcal{L}_{v}(h)=v^{\prime}(v(h)) \in \operatorname{Fun}(M)
$$

and therefore it is a Lie algebra homomorphism

$$
\left.\left[\mathcal{L}_{v^{\prime}}, \mathcal{L}_{v}\right](h)=\mathcal{L}_{\left[v^{\prime}\right.} v\right](h)
$$

This implies that we can extend the Lie derivative associated with a vectorfield to a Lie derivative associated with elements of $U \Xi$ by $^{3}$

$$
\mathcal{L}_{\xi \zeta}=\mathcal{L}_{\xi} \mathcal{L}_{\zeta}
$$

As in (2.12) we frequently use the notation

$$
\xi(h)=\mathcal{L}_{\xi}(h)
$$

for the action of $U \Xi$ on $F u n(M)$.

The map we want to deform is the usual pointwise multiplication map between functions

$$
\begin{aligned}
\mu: \quad \operatorname{Fun}(M) \otimes \operatorname{Fun}(M) & \rightarrow \operatorname{Fun}(M) \\
f \otimes g & \mapsto f g .
\end{aligned}
$$

To obtain $\mu_{\star}$ we first apply $\mathcal{F}^{-1}$ and then $\mu$

$$
\begin{aligned}
\mu_{\star}: \quad \operatorname{Fun}(M) \otimes F u n(M) & \stackrel{\mathcal{F}^{-1}}{\longrightarrow} \operatorname{Fun}(M) \otimes F u n(M) \stackrel{\mu}{\rightarrow} F u n(M) \\
f \otimes g & \mapsto \mathcal{F}^{-1}(f \otimes g) \mapsto \mu \circ \mathcal{F}^{-1}(f \otimes g) .
\end{aligned}
$$

\footnotetext{
${ }^{3}$ Since $\mathcal{L}_{\xi}$ is a differential operator, we have a map $\mathcal{L}: U \Xi \rightarrow$ Diff where Diff is the algebra of differential operators from $A$ to $A$. Notice that this map is neither surjective nor injective.
} 
This product is the $\star$-product

$$
f \star g \equiv \mu_{\star}(f, g):=\mu \circ \mathcal{F}^{-1}(f \otimes g) .
$$

We see that $\mu_{\star}=\mu \circ \mathcal{F}^{-1}$ is a bidifferential operator.

That the $\star$-product is associative follows from (2.9), see the theorem in Section 3.1 for the proof. This is only true because we have used $\mathcal{F}^{-1}$ and not $\mathcal{F}$ in (2.19). We also have

$$
f \star 1=f=1 \star f
$$

as a consequence of the normalisation condition (2.10). From (2.11) follows that

$$
f \star g=f g+\mathcal{O}(\lambda)
$$

We have thus deformed the commutative algebra of function $A \equiv F$ un $(M)$ into the noncommutative one

$$
A_{\star} \equiv \operatorname{Fun}_{\star}(M)
$$

We shall frequently use the notation (sum over $\alpha$ understood)

$$
\mathcal{F}=\mathrm{f}^{\alpha} \otimes \mathrm{f}_{\alpha} \quad, \quad \mathcal{F}^{-1}=\overline{\mathrm{f}}^{\alpha} \otimes \overline{\mathrm{f}}_{\alpha},
$$

so that

$$
f \star g:=\overline{\mathrm{f}}^{\alpha}(f) \overline{\mathrm{f}}_{\alpha}(g) .
$$

The elements $\mathrm{f}^{\alpha}, \mathrm{f}_{\alpha}, \overline{\mathrm{f}}^{\alpha}, \overline{\mathrm{f}}_{\alpha}$ live in $U \Xi$.

In order to get familiar with this notation we will rewrite equation (2.9) and its inverse,

$$
\left((\Delta \otimes i d) \mathcal{F}^{-1}\right) \mathcal{F}_{12}^{-1}=\left((i d \otimes \Delta) \mathcal{F}^{-1}\right) \mathcal{F}_{23}^{-1}
$$

as well as (2.10) and (2.11) using the notation (2.24), explicitly

$$
\begin{aligned}
\mathrm{f}^{\beta} \mathrm{f}_{1}^{\alpha} \otimes \mathrm{f}_{\beta} \mathrm{f}_{2}^{\alpha} \otimes \mathrm{f}_{\alpha} & =\mathrm{f}^{\alpha} \otimes \mathrm{f}^{\beta} \mathrm{f}_{\alpha_{1}} \otimes \mathrm{f}_{\beta} \mathrm{f}_{\alpha_{2}}, \\
\overline{\mathrm{f}}_{1}^{\alpha} \overline{\mathrm{f}}^{\beta} \otimes \overline{\mathrm{f}}_{2}^{\alpha} \overline{\mathrm{f}}_{\beta} \otimes \overline{\mathrm{f}}_{\alpha} & =\overline{\mathrm{f}}^{\alpha} \otimes \overline{\mathrm{f}}_{\alpha_{1}} \overline{\mathrm{f}}^{\beta} \otimes \overline{\mathrm{f}}_{\alpha_{2}} \overline{\mathrm{f}}_{\beta}, \\
\varepsilon\left(\mathrm{f}^{\alpha}\right) \mathrm{f}_{\alpha} & =1=\mathrm{f}^{\alpha} \varepsilon\left(\mathrm{f}_{\alpha}\right), \\
\mathcal{F}=\mathrm{f}^{\alpha} \otimes \mathrm{f}_{\alpha} & =1 \otimes 1+\mathcal{O}(\lambda) .
\end{aligned}
$$

\subsection{Examples of twists}

1) Consider the case $M=\mathbb{R}^{n}$ and the element

$$
\mathcal{F}=e^{-\frac{i}{2} \lambda \theta^{\mu \nu} \frac{\partial}{\partial x^{\mu}} \otimes \frac{\partial}{\partial x^{\nu}}}
$$


where $\theta^{\mu \nu}$ is an antisymmetric matrix of real numbers. The inverse of $\mathcal{F}$ is

$$
\mathcal{F}^{-1}=e^{\frac{i}{2} \lambda \theta^{\mu \nu} \frac{\partial}{\partial x^{\mu}} \otimes \frac{\partial}{\partial x^{\nu}}}
$$

Then we have

$$
(\Delta \otimes i d) \mathcal{F}=e^{-\frac{i}{2} \lambda \theta^{\mu \nu}\left(\frac{\partial}{\partial x^{\mu}} \otimes 1 \otimes \frac{\partial}{\partial x^{\nu}}+1 \otimes \frac{\partial}{\partial x^{\mu}} \otimes \frac{\partial}{\partial x^{\nu}}\right)}
$$

so that property (2.9) follows:

$$
\mathcal{F}_{12}(\Delta \otimes i d) \mathcal{F}=e^{-\frac{i}{2} \lambda \theta^{\mu \nu}\left(\frac{\partial}{\partial x^{\mu}} \otimes \frac{\partial}{\partial x^{\nu}} \otimes 1+\frac{\partial}{\partial x^{\mu}} \otimes 1 \otimes \frac{\partial}{\partial x^{\nu}}+1 \otimes \frac{\partial}{\partial x^{\mu}} \otimes \frac{\partial}{\partial x^{\nu}}\right)}=\mathcal{F}_{23}(i d \otimes \Delta) \mathcal{F}
$$

Property $(2.10)$ trivially holds. The $\star$-product that the twist $\mathcal{F}$ induces on the algebra of functions on $\mathbb{R}^{n}$ is the usual $\theta$-constant $\star$-product (Moyal-Weyl $\star$-product),

$$
(f \star g)(x)=\left.e^{\frac{i}{2} \lambda \theta^{\mu \nu} \frac{\partial}{\partial x^{\mu}} \frac{\partial}{\partial y^{\nu}}} f(x) g(y)\right|_{y \rightarrow x} .
$$

2) More in general on a smooth manifold $M$ consider a set of mutually commuting smooth vectorfields $\left\{X_{a}\right\}, a=1,2, \ldots s$. These vectorfields are globally defined on the manifold $M$ but can be zero outside a given open of $M$. Consider then

$$
\mathcal{F}=e^{\lambda \sigma^{a b} X_{a} \otimes X_{b}}
$$

where $\sigma^{a b}$ are arbitrary constants. The proof that $\mathcal{F}$ is a twist is the same as that of the first example.

In the case that $M$ is a Lie group (and more generally a quantum group) deformations of the form (2.33) appeared in [20]. See also [21] where a few examples that reproduce known $q$-deformed spaces are explicitly presented.

2a) A star product that implements the quantum plane commutation relation $x y=q y x$ $\left(q=e^{i \lambda}\right)$ can be obtained via the twist

$$
\mathcal{F}=e^{-\frac{i}{2} \lambda\left(x \frac{\partial}{\partial x} \otimes y \frac{\partial}{\partial y}-y \frac{\partial}{\partial y} \otimes x \frac{\partial}{\partial x}\right)} .
$$

Notice that the vectorfields $x \frac{\partial}{\partial x}$ and $y \frac{\partial}{\partial y}$ vanish at the origin. In the semiclassical limit we have a Poisson structure not a symplectic one.

2b) Consider the sphere $S^{2}$ and the usual polar coordinates $0 \leq \varphi<2 \pi, 0 \leq \vartheta \leq \pi$. Let $f(\varphi)$ and $l(\vartheta)$ be arbitrary smooth functions with support for example in $\left(-\frac{\pi}{4}, \frac{\pi}{4}\right)$ and $\left(\frac{\pi}{8}, \frac{3 \pi}{8}\right)$ respectively. Then

$$
\mathcal{F}=e^{\lambda f(\varphi) \frac{\partial}{\partial \varphi} \otimes l(\vartheta) \frac{\partial}{\partial \vartheta}}
$$

gives a well defined star product on the sphere. 
3) Twists are not necessarily related to commuting vectorfields. For example consider on a smooth manifold $M$ four vectorfields $H, E, A, B$, that satisfy the Lie algebra relations

$$
\begin{aligned}
& {[H, E]=2 E, \quad[H, A]=\alpha A, \quad[H, B]=\beta B, \quad \alpha+\beta=2,} \\
& {[A, B]=E, \quad[E, A]=0, \quad[E, B]=0 \text {. }}
\end{aligned}
$$

Then the element

$$
\mathcal{F}=e^{\frac{1}{2} H \otimes \ln (1+\lambda E)} e^{\lambda A \otimes B \frac{1}{1+\lambda E}}
$$

is a twist and gives a well defined $\star$-product on the algebra of functions on $M$. These twists are known as extended Jordanian deformations [24]. Jordanian deformations $[22,23]$ are obtained setting $A=B=0$ (and keeping the relation $[H, E]=2 E$ ).

\subsubsection{Deformed Superspace}

Consider the superspace $\mathbb{R}^{m \mid n}$ with coordinates $\left(x^{\mu}, \theta^{\alpha}\right) \equiv Z^{A}$ and partial derivatives $\left(\partial_{\mu}, \partial_{\alpha}\right) \equiv \partial_{A}$ that satisfy the following (anti-)commutation relations

$$
\left[Z^{A}, Z^{B}\right]_{ \pm}=0, \partial_{A} Z^{B}=\delta_{A}^{B}
$$

A generic derivation is of the form $\chi=f^{A}(Z) \partial_{A}$, where $f^{A}(Z)$ are functions on superspace. Consider a set $\left\{\chi_{a}, \chi_{\varepsilon}\right\} \equiv\left\{\chi_{I}\right\}$ of even derivations $\chi_{a}$ and of odd derivations $\chi_{\varepsilon}$ that are mutually (anti-)commuting,

$$
\left[\chi_{I}, \chi_{J}\right]_{ \pm}=0
$$

for instance one can consider the derivativations $\left\{\chi_{I}\right\}=\left\{\partial_{\mu}, \partial_{\alpha}\right\}$, or the derivations $\left\{\chi_{I}\right\}=\left\{\frac{\partial}{\partial x^{1}}, \theta^{1} \frac{\partial}{\partial \theta^{1}}, \theta^{2} \frac{\partial}{\partial \theta^{2}}, \frac{\partial}{\partial \theta^{3}}, \theta^{4} \frac{\partial}{\partial x^{2}}\right\}$ (if $m \geq 2$ and $n \geq 4$ ).

The universal enveloping superalgebra of the Lie superalgebra (2.38) is as usual the algebra $\mathcal{U}$ over $\mathbb{C}$ generated by the elements $\chi_{I}$ modulo the relations $(2.38)$. The algebra $\mathcal{U}$ becomes a Hopf superalgebra by defining on the generators the following grade preserving coproduct and antipode, and the following counit:

$$
\Delta\left(\chi_{I}\right):=\chi_{I} \otimes 1+1 \otimes \chi_{I}, \quad S\left(\chi_{I}\right):=-\chi_{I}, \quad \varepsilon\left(\chi_{I}\right):=0,
$$

where the tensorproduct $\otimes$ is over $\mathbb{C}$. The multiplication in $\mathcal{U} \otimes \mathcal{U}$ is defined as follows for homogeneous elements $\xi, \zeta, \xi^{\prime}, \zeta^{\prime} \in \mathcal{U}$ (of even or odd degree $|\xi|,|\zeta|,\left|\xi^{\prime}\right|,\left|\zeta^{\prime}\right|$ respectively):

$$
(\xi \otimes \zeta)\left(\xi^{\prime} \otimes \zeta^{\prime}\right)=(-1)^{|\zeta|\left|\xi^{\prime}\right|} \xi \xi^{\prime} \otimes \zeta \zeta^{\prime} .
$$

The antipode is extended to all elements of $\mathcal{U}$ by requiring it to be linear and graded antimultiplicative; the coproduct is linear and multiplicative (the grading being already present in (2.39)); the counit is linear and multiplicative:

$$
\Delta(\xi \zeta)=\Delta(\xi) \Delta(\eta) \quad, \quad S(\xi \zeta)=(-1)^{|\zeta||\xi|} S(\zeta) S(\xi) \quad, \quad \varepsilon(\xi \zeta)=\varepsilon(\xi) \varepsilon(\zeta) .
$$


We refer to [50] for a concise treatment of Hopf superalgebras.

Consider the even element in $\mathcal{U}[[\lambda]] \otimes \mathcal{U}[[\lambda]]$ given by

$$
\mathcal{F}:=e^{\lambda \sigma^{I J} \chi_{I} \otimes \chi_{J}}=e^{\lambda \sigma^{a a^{\prime}}} \chi_{a} \otimes \chi_{a^{\prime}}+\lambda \sigma^{\varepsilon \varepsilon^{\prime}} \chi_{\varepsilon} \otimes \chi_{\varepsilon^{\prime}},
$$

where $\left\{\sigma_{I J}\right\} \equiv\left\{\sigma_{a a^{\prime}}, \sigma_{\varepsilon \varepsilon^{\prime}}\right\}$ are arbitrary constants (C्C-numbers). In order to check that $\mathcal{F}$ is a twist as defined in Definition 1 we observe that $\mathcal{F}_{12}=e^{\lambda \sigma^{I J}} \chi_{I} \otimes \chi_{J} \otimes 1=e^{\lambda \sigma^{I J}} \chi_{I} \otimes \chi_{J} \otimes 1$, and that

$$
(\Delta \otimes i d) \mathcal{F}=e^{\lambda \sigma^{I J}\left(\chi_{I} \otimes 1+1 \otimes \chi_{I}\right) \otimes \chi_{J}} .
$$

This last relation holds because $\Delta \otimes i d: \mathcal{U} \otimes \mathcal{U} \rightarrow \mathcal{U} \otimes \mathcal{U} \otimes \mathcal{U}$ is multiplicative (the product in $\mathcal{U} \otimes \mathcal{U} \otimes \mathcal{U}$ is given by $\left.(\xi \otimes \zeta \otimes \eta)\left(\xi^{\prime} \otimes \zeta^{\prime} \otimes \eta^{\prime}\right)=(-1)^{|\zeta+\eta|\left|\xi^{\prime}\right|+|\eta|\left|\zeta^{\prime}\right|} \xi \xi^{\prime} \otimes \zeta \zeta^{\prime} \otimes \eta \eta^{\prime}\right)$. Finally

$$
\mathcal{F}_{12}(\Delta \otimes i d) \mathcal{F}=e^{\lambda \sigma^{I J} \chi_{I} \otimes \chi_{J} \otimes 1+\lambda \sigma^{I J}\left(\chi_{I} \otimes 1+1 \otimes \chi_{I}\right) \otimes \chi_{J}}
$$

because the arguments of the exponentials are even elements of $\mathcal{U} \otimes \mathcal{U} \otimes \mathcal{U}$ whose commutator vanishes. One similarly computes $\mathcal{F}_{23}(i d \otimes \mathcal{F})$.

An associative $\star$-product on superspace is then defined by

$$
\begin{aligned}
g \star h & :=\mu \circ \mathcal{F}^{-1}(g \otimes h) \\
& =(-1)^{\left|\overline{\mathrm{f}}_{\alpha}\right||g|} \overline{\mathrm{f}}^{\alpha}(g) \overline{\mathrm{f}}_{\alpha}(h) .
\end{aligned}
$$

Associativity depends only on property (2.28) and not on the specific example of twists (2.41). Associativity is explicitly proven in Appendix A.3.

As particular cases of this construction we obtain the non anti-commutative superspaces considered in [51]. For twists on superspace see also [52] and references therein.

\subsection{The deformed Hopf algebra $U \Xi^{\mathcal{F}}$}

Another deformation via the action of $\mathcal{F}$ leads to a new Hopf algebra

$$
\left(U \Xi^{\mathcal{F}}, \cdot, \Delta^{\mathcal{F}}, S^{\mathcal{F}}, \varepsilon^{\mathcal{F}}\right)=\left(U \Xi, \cdot \Delta^{\mathcal{F}}, S^{\mathcal{F}}, \varepsilon\right)
$$

As algebras $U \Xi^{\mathcal{F}}=U \Xi$ and they also have the same counit $\varepsilon^{\mathcal{F}}=\varepsilon$. The new coproduct $\Delta^{\mathcal{F}}$ is given by

$$
\begin{aligned}
\Delta^{\mathcal{F}}: \quad U \Xi^{\mathcal{F}} & =U \Xi \stackrel{\Delta}{\longrightarrow} U \Xi \otimes U \Xi \stackrel{\text { Conj }_{\mathcal{F}}}{\longrightarrow} U \Xi \otimes U \Xi=U \Xi^{\mathcal{F}} \otimes U \Xi^{\mathcal{F}} \\
\xi & \mapsto \Delta(\xi) \mapsto \Delta^{\mathcal{F}}(\xi)=\mathcal{F} \Delta(\xi) \mathcal{F}^{-1} .
\end{aligned}
$$

We deform the antipode, a map from $U \Xi$ to $U \Xi$, using an invertible element $\chi$ of $U \Xi$ defined as follows ${ }^{4}$

$$
\chi:=\mathrm{f}^{\alpha} S\left(\mathrm{f}_{\alpha}\right) \quad, \quad \chi^{-1}=S\left(\overline{\mathrm{f}}^{\alpha}\right) \overline{\mathrm{f}}_{\alpha} .
$$

\footnotetext{
${ }^{4}$ See Appendix A.1 for a proof that $\chi \chi^{-1}=\chi^{-1} \chi=1$.
} 
The definition of the new antipode is

$$
S^{\mathcal{F}}(\xi)=\chi S(\xi) \chi^{-1}
$$

We follow the same steps as in Subsection (2.1) to show that $U \Xi^{\mathcal{F}}=\left(U \Xi^{\mathcal{F}}, \cdot, \Delta^{\mathcal{F}}, S^{\mathcal{F}}, \varepsilon\right)$ is a Hopf algebra.

That $\Delta^{\mathcal{F}}$ and $\varepsilon$ are algebra homomorphisms and that $S^{\mathcal{F}}$ is an antialgebra homomorphism follows immediately from the definition

$$
\begin{aligned}
\Delta^{\mathcal{F}}(\xi \zeta) & =\Delta^{\mathcal{F}}(\xi) \Delta^{\mathcal{F}}(\zeta) \\
\varepsilon^{\mathcal{F}}(\xi \zeta) & =\varepsilon^{\mathcal{F}}(\xi) \varepsilon^{\mathcal{F}}(\zeta) \\
S^{\mathcal{F}}(\xi \zeta) & =S^{\mathcal{F}}(\zeta) S^{\mathcal{F}}(\xi)
\end{aligned}
$$

We have now to show that $\Delta^{\mathcal{F}}$ and $S^{\mathcal{F}}$ fulfill the additional conditions (2.6), and therefore that $\left(U \Xi^{\mathcal{F}}, \cdot, \Delta^{\mathcal{F}}, S^{\mathcal{F}}, \varepsilon\right)$ is a Hopf algebra. This is done in Appendix A.1.

The new Hopf algebra $U \Xi^{\mathcal{F}}$ is triangular, i.e., there exists an invertible element $\mathcal{R} \in U \Xi^{\mathcal{F}} \otimes U \Xi^{\mathcal{F}}$ (called universal $\mathcal{R}$-matrix) such that, for all $\xi \in U \Xi$,

$$
\begin{gathered}
\Delta^{\mathcal{F} o p}(\xi)=\mathcal{R} \Delta^{\mathcal{F}}(\xi) \mathcal{R}^{-1} \\
\left(\Delta^{\mathcal{F}} \otimes i d\right) \mathcal{R}=\mathcal{R}_{13} \mathcal{R}_{23}, \quad\left(i d \otimes \Delta^{\mathcal{F}}\right) \mathcal{R}=\mathcal{R}_{13} \mathcal{R}_{12} \\
\mathcal{R}_{21}=\mathcal{R}^{-1}
\end{gathered}
$$

where $\mathcal{R}_{21}=\sigma(\mathcal{R}) \in U \Xi^{\mathcal{F}} \otimes U \Xi^{\mathcal{F}}$, with $\sigma$ the flip map, $\sigma(\xi \otimes \zeta)=\zeta \otimes \xi$. The two equations in (2.51) take value in $U \Xi \otimes U \Xi \otimes U \Xi$, and $\mathcal{R}_{12}=\mathcal{R} \otimes 1, \mathcal{R}_{23}=1 \otimes \mathcal{R}$, while $\mathcal{R}_{13} \in U \Xi \otimes U \Xi \otimes U \Xi$ has the unit 1 in the middle factor. Defining

$$
\mathcal{R}:=\mathcal{F}_{21} \mathcal{F}^{-1}
$$

it can be shown that equations (2.50), (2.51), (2.52) are fulfilled. The cocycle condition of $\mathcal{F}$ was in this context only needed to prove $(2.51)^{5}$. In the sequel we use the notation

$$
\mathcal{R}=R^{\alpha} \otimes R_{\alpha} \quad, \quad \mathcal{R}^{-1}=\bar{R}^{\alpha} \otimes \bar{R}_{\alpha}
$$

Using the notation introduced in (2.24) we obtain

$$
\mathcal{R}=R^{\alpha} \otimes R_{\alpha}=\mathrm{f}_{\alpha} \overline{\mathrm{f}}^{\beta} \otimes \mathrm{f}^{\alpha \overline{\mathrm{f}}_{\beta}}, \quad \mathcal{R}^{-1}=\bar{R}^{\alpha} \otimes \bar{R}_{\alpha}=\mathrm{f}^{\alpha \overline{\mathrm{f}}_{\beta}} \otimes \mathrm{f}_{\alpha} \overline{\mathrm{f}}^{\beta} .
$$

\footnotetext{
${ }^{5}$ We refer to [48] (p. 56), see also [49] (p.130), for a proof of (2.51) and for an introduction to twists and their relations to Hopf algebra deformations.
} 


\section{Representations}

\subsection{Module Algebras}

Having a Hopf algebra, its modules are certainly of interest in physics and mathematics. They are the representations of the Hopf algebra. Here we show that to a module algebra $\mathcal{A}$ of the Hopf algebra $U \Xi$ there corresponds a module algebra $\mathcal{A}_{\star}$ of the deformed Hopf algebra $U \Xi^{\mathcal{F}}$.

A module algebra $\mathcal{A}$ is a module $\mathcal{A}$ on which $U \Xi$ acts which in addition has an algebra structure that is compatible with the action of $U \Xi$, for all $\xi \in U \Xi$ and $a, b \in \mathcal{A}$,

$$
\xi(a b)=\mu \circ \Delta(\xi)(a \otimes b)=\xi_{1}(a) \xi_{2}(b) \quad, \quad \xi(1)=\varepsilon(\xi) 1
$$

(where 1 is the unit in $\mathcal{A}$ ).

We recall a basic theorem concerning representations of twisted Hopf algebras. Given a twist $\mathcal{F} \in U \Xi \otimes U \Xi$, we can construct a deformed algebra $\mathcal{A}_{\star}$. The algebra $\mathcal{A}_{\star}$ has the same vector space structure as $\mathcal{A}$ and the action of $U \Xi^{\mathcal{F}}$ on $\mathcal{A}_{\star}$ is the action of $U \Xi$ on $\mathcal{A}$. The product in $\mathcal{A}_{\star}$ is defined by

$$
a \star b=\mu \circ \mathcal{F}^{-1}(a \otimes b)=\overline{\mathrm{f}}^{\alpha}(a) \overline{\mathrm{f}}_{\alpha}(b)
$$

in accordance with formula (2.20). Compatibility between the action of $U \Xi^{\mathcal{F}}$ and the product in $\mathcal{A}_{\star}$ demands

$$
\xi(a \star b)=\xi_{1_{\mathcal{F}}}(a) \star \xi_{2_{\mathcal{F}}}(b),
$$

where we used the notation $\Delta^{\mathcal{F}}(\xi)=\xi_{1_{\mathcal{F}}} \otimes \xi_{2_{\mathcal{F}}}$.

In order to prove associativity of the new product we use (2.28) and compute:

$$
\begin{aligned}
(a \star b) \star c & =\overline{\mathrm{f}}^{\alpha}\left(\overline{\mathrm{f}}^{\beta}(a) \overline{\mathrm{f}}_{\beta}(b)\right) \overline{\mathrm{f}}_{\alpha}(c)=\left(\overline{\mathrm{f}}_{1}^{\alpha} \overline{\mathrm{f}}^{\beta}\right)(a)\left(\overline{\mathrm{f}}_{2}^{\alpha} \overline{\mathrm{f}}_{\beta}\right)(b) \overline{\mathrm{f}}_{\alpha}(c)=\overline{\mathrm{f}}^{\alpha}(a)\left(\overline{\mathrm{f}}_{\alpha_{1}} \overline{\mathrm{f}}^{\beta}\right)(b)\left(\overline{\mathrm{f}}_{\alpha_{2}} \overline{\mathrm{f}}_{\beta}\right)(c) \\
& =\overline{\mathrm{f}}^{\alpha}(a) \overline{\mathrm{f}}_{\alpha}\left(\overline{\mathrm{f}}^{\beta}(b) \overline{\mathrm{f}}_{\beta}(c)\right)=a \star(b \star c) .
\end{aligned}
$$

We still have to prove (3.2):

$\xi(a \star b)=\xi\left(\mu \circ \mathcal{F}^{-1}(a \otimes b)\right)=\mu \circ \Delta(\xi) \circ \mathcal{F}^{-1}(a \otimes b)=\mu \circ \mathcal{F}^{-1} \circ \Delta^{\mathcal{F}}(\xi)(a \otimes b)=\xi_{1_{\mathcal{F}}}(a) \star \xi_{2 \mathcal{F}}(b)$.

Notice also that if $\mathcal{A}$ has a unit element 1 , then $1 \star a=a \star 1$ follows from the normalization condition property $(2.10)$ of the twist $\mathcal{F}$.

\subsection{Examples of Module algebras}

We now apply this construction to the $U \Xi$-module algebras $A$ and $U \Xi$. In both cases the action of $U \Xi$ on the corresponding module algebra is given by the Lie derivative. 


\section{Algebra of noncommutative functions $A_{\star}$}

We start with the $U \Xi$-module algebra of functions $\mathcal{A}=A=F u n(M)$, and we obtain the algebra $A_{\star} \equiv F_{n_{\star}}(M)$ with the $\star$-product already introduced in (2.20). The algebra $A_{\star}$, according to Section 3.1 is a left $U \Xi^{\mathcal{F}}$-module algebra. In particular, vectorfields $u \in \Xi \subset U \Xi^{\mathcal{F}}$ act according to the deformed Leibniz rule

$$
u(h \star g)=u_{1_{\mathcal{F}}}(h) \star u_{2_{\mathcal{F}}}(g),
$$

where

$$
\Delta^{\mathcal{F}}(u)=u_{1_{\mathcal{F}}} \otimes u_{2_{\mathcal{F}}}=\mathrm{f}^{\alpha} u \overline{\mathrm{f}}^{\beta} \otimes \mathrm{f}_{\alpha} \overline{\mathrm{f}}_{\beta}+\mathrm{f}^{\alpha} \overline{\mathrm{f}}^{\beta} \otimes \mathrm{f}_{\alpha} u \overline{\mathrm{f}}_{\beta} .
$$

The algebra $U \Xi_{\star}$

We next consider the case $\mathcal{A}=U \Xi$. This is a module algebra with respect to the Hopf algebra $U \Xi$. The action of $U \Xi$ on $U \Xi$ is given by the extended Lie derivative (adjoint action): the action of $\mathcal{L}_{u}$ on $v$ is just the Lie bracket $\mathcal{L}_{u}(v)=[u v]$; the action of $U \Xi$ on $\Xi$ is obtained from the action of vectorfields by definining $\mathcal{L}_{\xi \zeta}=\mathcal{L}_{\xi} \mathcal{L}_{\zeta}$ (where composition of the actions $\mathcal{L}_{\xi}$ and $\mathcal{L}_{\zeta}$ is understood); finally the action of $U \Xi$ on $U \Xi$ is obtained from the known Leibniz rule $\mathcal{L}_{u}(v z)=\mathcal{L}_{u}(v) z+v \mathcal{L}_{u}(z)$, that implies $\mathcal{L}_{\xi}(\zeta \eta)=\mathcal{L}_{\xi_{1}}(\zeta) \mathcal{L}_{\xi_{2}}(\eta)$.

The deformed algebra $U \Xi_{\star}$ equals $U \Xi$ as a vectorspace, but it has the deformed product

$$
\begin{aligned}
\star: U \Xi \otimes U \Xi & \rightarrow U \Xi \\
(\xi, \zeta) & \mapsto \xi \star \zeta:=\overline{\mathrm{f}}^{\alpha}(\xi) \overline{\mathrm{f}}_{\alpha}(\zeta)
\end{aligned}
$$

where $\overline{\mathrm{f}}^{\alpha}(\xi)$, (and $\left.\overline{\mathrm{f}}_{\alpha}(\zeta)\right)$ is another notation for the Lie derivative $\mathcal{L}_{\overline{\mathrm{f}}^{\alpha}}(\xi)$, (and $\mathcal{L}_{\overline{\mathrm{f}}_{\alpha}}(\zeta)$ ). The Hopf algebra $U \Xi^{\mathcal{F}}$ acts on $U \Xi_{\star}$, and compatibility with the $\star$-product of $U \Xi_{\star}$ is

$$
\xi(\zeta \star \eta)=\xi_{1_{\mathcal{F}}}(\zeta) \star \xi_{2_{\mathcal{F}}}(\eta) .
$$

This way we have obtained from the theorem in Section 3.1 the algebra $U \Xi_{\star}$. We will show in Section 3.3 that it is a Hopf algebra.

In $U \Xi_{\star}$ we consider the deformed commutator of the vectorfields $u, v \in \Xi$,

$$
[u, v]_{\star}:=u \star v-\bar{R}^{\alpha}(v) \star \bar{R}_{\alpha}(u) .
$$

This commutator closes in $\Xi$ :

$$
\begin{aligned}
u \star v-\bar{R}^{\alpha}(v) \star \bar{R}_{\alpha}(u) & =\overline{\mathrm{f}}^{\gamma}(u) \overline{\mathrm{f}}_{\gamma}(v)-\overline{\mathrm{f}}^{\gamma}\left(\bar{R}_{\alpha}(v)\right) \overline{\mathrm{f}}_{\gamma}\left(\bar{R}^{\alpha}(u)\right) \\
& =\overline{\mathrm{f}}^{\gamma}(u) \overline{\mathrm{f}}_{\gamma}(v)-\overline{\mathrm{f}}^{\gamma} \mathrm{f}^{\alpha} \overline{\mathrm{f}}_{\beta}(v) \overline{\mathrm{f}}_{\gamma} \mathrm{f}_{\alpha} \overline{\mathrm{f}}^{\beta}(u) \\
& =\overline{\mathrm{f}}^{\gamma}(u) \overline{\mathrm{f}}_{\gamma}(v)-\overline{\mathrm{f}}_{\gamma}(v) \overline{\mathrm{f}}^{\gamma}(u) \\
& =\left[\overline{\mathrm{f}}^{\gamma}(u), \overline{\mathrm{f}}_{\gamma}(v)\right],
\end{aligned}
$$


(the first line uses the definition of the $\star$-product, the second line the definition of the $\mathcal{R}$-matrix, $\mathcal{R}^{-1}=\bar{R}^{\alpha} \otimes \bar{R}_{\alpha}=\mathrm{f}^{\alpha} \overline{\mathrm{f}}_{\beta} \otimes \mathrm{f}_{\alpha} \overline{\mathrm{f}}^{\beta}$ as introduced in Section 2.4. The third line uses $\mathcal{F}^{-1} \mathcal{F}=1$ ). The last term is a a sum (over $\gamma$ ) of undeformed commutators between the vectorfields $\overline{\mathrm{f}}^{\gamma}(u)$ and $\overline{\mathrm{f}}_{\gamma}(v)$, and therefore $[u, v]_{\star} \in \Xi$.

We denote by $\Xi_{\star}$ the linear space of vectorfields $\Xi$ equipped with the multiplication

$$
\begin{aligned}
{[,]_{\star}: \quad \Xi \times \Xi } & \rightarrow \Xi \\
(u, v) & \mapsto[u, v]_{\star} .
\end{aligned}
$$

this way $\Xi_{\star}$ becomes a deformed Lie algebra. The elements of $\Xi_{\star}$ we call $\star$-vectorfields. It is easy to see that the bracket $[,]_{\star}$ has the $\star$-antisymmetry property

$$
[u, v]_{\star}=-\left[\bar{R}^{\alpha}(v), \bar{R}_{\alpha}(u)\right]_{\star} .
$$

This can be shown as follows

$$
[u, v]_{\star}=\left[\overline{\mathrm{f}}^{\alpha}(u), \overline{\mathrm{f}}_{\alpha}(v)\right]=-\left[\overline{\mathrm{f}}_{\alpha}(v), \overline{\mathrm{f}}^{\alpha}(u)\right]=-\left[\bar{R}^{\alpha}(v), \bar{R}_{\alpha}(u)\right]_{\star} .
$$

We recall that $\mathcal{R}^{-1}=\bar{R}^{\alpha} \otimes \bar{R}_{\alpha}=\mathcal{F F}_{21}^{-1} \in U \Xi \otimes U \Xi$.

A $\star$-Jacoby identity can be proven as well

$$
\left[u,[v, z]_{\star}\right]_{\star}=\left[[u, v]_{\star}, z\right]_{\star}+\left[\bar{R}^{\alpha}(v),\left[\bar{R}_{\alpha}(u), z\right]_{\star}\right]_{\star} .
$$

A direct proof of the $\star$-Jacobi identity can be found in Appendix A.2.

Finally we notice that any sum of products of vectorfields in $U \Xi$ can be rewritten as sum of $\star$-products of vectorfields via the formula $u v=\mathrm{f}^{\alpha}(u) \star \mathrm{f}_{\alpha}(v)$, and therefore $\star$-vectorfields generate the algebra.

Indeed we have proven, see [44], that $U \Xi_{\star}$ is the universal enveloping algebra of $\Xi_{\star}$.

\section{3 $U \Xi_{\star}$ is a Hopf algebra}

We have seen that $U \Xi$ can be equipped with the usual Hopf algebra structure $(U \Xi, \cdot, \Delta, S, \varepsilon)$ or with the twisted Hopf algebra $\left(U \Xi^{\mathcal{F}}, \cdot, \Delta^{\mathcal{F}}, S^{\mathcal{F}}, \varepsilon\right)$ or with a new product $U \Xi_{\star}=$ $(U \Xi, \star)$. It turns out that $U \Xi_{\star}$ has also a natural Hopf algebra structure,

$$
\left(U \Xi_{\star}, \star, \Delta_{\star}, S_{\star}, \varepsilon_{\star}\right)
$$

We describe it by giving the coproduct, the inverse of the antipode and the counit on the generators $u$ of $U \Xi_{\star}$ :

$$
\begin{gathered}
\Delta_{\star}(u)=u \otimes 1+X_{\bar{R}^{\alpha}} \otimes \bar{R}_{\alpha}(u) \\
S_{\star}^{-1}(u)=-\bar{R}^{\alpha}(u) \star X_{\bar{R}_{\alpha}} .
\end{gathered}
$$




$$
\varepsilon_{\star}(u)=\varepsilon(u)=0,
$$

where, for all $\xi \in U \Xi, X_{\xi}=\overline{\mathrm{f}}^{\alpha} \xi \chi S^{-1}\left(\overline{\mathrm{f}}_{\alpha}\right)$. The map $X: U \Xi \rightarrow U \Xi$ is invertible and it can be shown [53], that its inverse $X^{-1}$ is

$$
X^{-1}=\overline{\mathrm{f}}^{\alpha}(\xi) \overline{\mathrm{f}}_{\alpha}=: D(\xi) .
$$

In principle one could directly check that (3.12)-(3.14) define a bona fide Hopf algebra. Another way [44] is to show that the Hopf algebra $U \Xi_{\star}$ is isomorphic to the Hopf algebra $U \Xi^{\mathcal{F}}$. The isomorphism is given by the map $D$ :

$$
\begin{aligned}
& D(\xi \star \zeta)=D(\xi) D(\zeta), \\
& \Delta_{\star}=\left(D^{-1} \otimes D^{-1}\right) \circ \Delta^{\mathcal{F}} \circ D, \\
& S_{\star}=D^{-1} \circ S^{\mathcal{F}} \circ D .
\end{aligned}
$$

In particular, since $U \Xi^{\mathcal{F}}$ is a triangular Hopf algebra, also $U \Xi_{\star}$ is a triangular Hopf algebra. Its $R$-matrix is

$$
\mathcal{R}_{\star}=\left(D^{-1} \otimes D^{-1}\right)(\mathcal{R}) \quad, \quad \mathcal{R}_{\star}=R_{\star}^{\alpha} \otimes R_{\star \alpha}=X_{R^{\alpha}} \otimes X_{R_{\alpha}} .
$$

Explicitly we have

$$
\begin{gathered}
\Delta_{\star}^{o p}(\xi)=\mathcal{R}_{\star} \star \Delta_{\star}(\xi) \star \mathcal{R}_{\star}^{-1} \\
\left(\Delta_{\star} \otimes i d\right) \mathcal{R}_{\star}=\mathcal{R}_{\star 13} \star \mathcal{R}_{\star 23}, \quad\left(i d \otimes \Delta_{\star}\right) \mathcal{R}_{\star}=\mathcal{R}_{\star 13} \star \mathcal{R}_{\star 12}, \\
\mathcal{R}_{\star 21}=\mathcal{R}_{\star}^{-1},
\end{gathered}
$$

where $\mathcal{R}_{\star}^{-1}$ is the $\star$-inverse of $\mathcal{R}_{\star}$, i.e., $\mathcal{R}_{\star}^{-1} \star \mathcal{R}_{\star}=\mathcal{R}_{\star} \star \mathcal{R}_{\star}^{-1}=1 \otimes 1$.

Summarizing we have encountered the Hopf algebras

$$
(U \Xi, \cdot \Delta, S, \varepsilon), \quad\left(U \Xi^{\mathcal{F}}, \cdot \Delta^{\mathcal{F}}, S^{\mathcal{F}}, \varepsilon\right), \quad\left(U \Xi_{\star}, \star, \Delta_{\star}, S_{\star}, \varepsilon\right) .
$$

The first is cocommutative, the second is triangular and is obtained twisting the first, the third is triangular and isomorphic to the second. The remarkable fact about $U \Xi_{\star}$ is the Leibniz rule for vectorfields (3.12). We have that $\bar{R}_{\alpha}(u)$ is again a vectorfield so that

$$
\Delta_{\star}\left(\Xi_{\star}\right) \subset \Xi_{\star} \otimes 1+U \Xi_{\star} \otimes \Xi_{\star}
$$

This is a fundamental property for the construction of a deformed differential calculus à la Woronowicz [40]. Note that the coproduct $\Delta^{\mathcal{F}}(u)$ does not have this property, as can be seen explicitly from (3.4). It is interesting to note that a Hopf algebra with comultiplication structure (3.4) is isomorphic to a Hopf algebra with comultiplication structure (3.23). In order to establish a gravity theory which is invariant with respect to deformed infinitesimal diffeomorphisms we will consider module algebras with respect to $U \Xi_{\star}$ and not with respect to $U \Xi^{\mathcal{F}}$. 


\section{Representations of deformed infinitesimal diffeo- morphisms}

In Section 3 we have constructed the Hopf algebra $U \Xi_{\star}$. Since $U \Xi_{\star}$ and $U \Xi^{\mathcal{F}}$ are isomorphic as Hopf algebras, any $U \Xi^{\mathcal{F}}$-module has automatically a $U \Xi_{\star}$-module structure. In particular $A_{\star}$ and $U \Xi_{\star}$ are also $U \Xi_{\star}$-module algebras.

The action $\mathcal{L}^{\star}$ of $U \Xi_{\star}$ on $A_{\star}$ is given by combining the usual action (Lie derivative $\mathcal{L})$ with the twist $\mathcal{F}$

$$
\mathcal{L}_{\xi}^{\star}(h):=\mathcal{L}_{\overline{\mathrm{f}}^{\alpha}{ }^{\alpha}(\xi)}\left(\mathcal{L}_{\overline{\mathrm{f}}_{\alpha}}(h)\right),
$$

or equivalently, recalling that $D(\xi)=\overline{\mathrm{f}}^{\alpha}(\xi) \overline{\mathrm{f}}_{\alpha}$, we see that

$$
\mathcal{L}_{\xi}^{\star}:=\mathcal{L}_{D(\xi)} .
$$

Similarly for the action of $U \Xi_{\star}$ on $U \Xi_{\star}$, that we also denote by $\mathcal{L}^{\star}$,

$$
\mathcal{L}_{\xi}^{\star}(\zeta):=\mathcal{L}_{\overline{\mathrm{f}}^{\alpha}(\xi)}\left(\mathcal{L}_{\overline{\mathrm{f}}_{\alpha}}(\zeta)\right)=\overline{\mathrm{f}}^{\alpha}(\xi)\left(\overline{\mathrm{f}}_{\alpha}(\zeta)\right)
$$

It is easy to see that these actions are well defined: $\mathcal{L}_{\xi}^{\star} \circ \mathcal{L}_{\zeta}^{\star}=\mathcal{L}_{\xi \star \zeta}^{\star}$, for example we find ${ }^{6}$

$$
\mathcal{L}_{\zeta}^{\star}\left(\mathcal{L}_{\xi}^{\star}(h)\right)=\mathcal{L}_{\zeta}^{\star}((D \xi)(h))=(D \xi)(D \zeta)(h)=D(\xi \star \zeta)(h)=\mathcal{L}_{\xi \star \zeta}^{\star}(h)
$$

where we used (3.16). Compatibility with the $\star$-product in $A_{\star}$ is also easily proven,

$$
\begin{aligned}
\mathcal{L}_{\xi}^{\star}(h \star g) & =\mathcal{L}_{D \xi}(h \star g)=(D \xi)(h \star g)=(D \xi)_{1_{\mathcal{F}}}(h) \star(D \xi)_{2_{\mathcal{F}}}(g)=D\left(\xi_{1_{\star}}\right)(h) \star D\left(\xi_{2_{\star}}\right)(g) \\
& =\mathcal{L}_{\xi_{1 \star}}^{\star}(h) \star \mathcal{L}_{\xi_{2 \star}}^{\star}(g)
\end{aligned}
$$

where we used (3.17). One proceeds similarly for the action $\mathcal{L}^{\star}$ of $U \Xi_{\star}$ on $U \Xi_{\star}$. The proofs that this action is well defined and that it is compatible with the $\star$-product in $U \Xi_{\star}$, are exactly the same as in (4.4) and (4.5), just substitute $h, g \in A_{\star}$ with $\zeta, \eta \in U \Xi_{\star}$. We here notice in particular that the $\star$-Lie derivative of a vectorfield on a vectorfield gives the $\star$-Lie bracket,

$$
\mathcal{L}_{u}^{\star}(v)=[u, v]_{\star} .
$$

Morover it can be shown that the $\star$-Lie derivative of $U \Xi_{\star}$ on $U \Xi_{\star}$ equals the $\star$-adjoint action, $\mathcal{L}_{\xi}^{\star}(\zeta)=a d_{\xi}^{\star}(\zeta) \equiv \xi_{1_{\star}} \star \zeta \star S_{\star}\left(\xi_{2 \star}\right)$. In particular the $\star$-commutator $[u, v]_{\star}$ is just the $\star$-adjoint action of $u$ on $v$.

\footnotetext{
${ }^{6}$ In [45], [14], [46], we have $\theta^{\mu \nu}$-constant noncommutativity and differential operators $X_{u}^{\star}$ that satisfy $X_{u}^{\star} X_{v}^{\star}=X_{u v}^{\star}$, the relation between $X_{u}^{\star}$ and $\mathcal{L}_{u}^{\star}$ (for the $\theta^{\mu \nu}$-constant case) is $\left(X_{u}^{\star} \star g\right)=u(g)=\mathcal{L}_{X_{u}}^{\star}(g)$.
} 


\subsection{Tensorfields}

Our main interest in this subsection is the deformed algebra of tensorfields. We recall that tensorfields on a smooth manifold can be described as elements in ${ }^{7}$

$$
\Omega \otimes \Omega \otimes \ldots \Omega \otimes \Xi \otimes \Xi \otimes \ldots \Xi
$$

where $\otimes$ here stands for $\otimes_{A}$. Functions are in particular type $(0,0)$-tensorfields and the tensorproduct between a function and another tensorfield is as usual not explicitly written. The tensorproduct is an associative product. This in particular implies $\tau \otimes h \tau^{\prime}=$ $\tau h \otimes \tau^{\prime}$ and $h\left(\tau \otimes \tau^{\prime}\right)=(h \tau) \otimes \tau^{\prime}$. Tensorfields are a $U \Xi$ module, the action of $U \Xi$ on $\mathcal{T}$ is obtained via the Lie derivative on tensorfields, that extends to a map $\mathcal{L}: U \Xi \otimes \mathcal{T} \rightarrow \mathcal{T}$. For example $\mathcal{L}_{u v}(\tau)=\mathcal{L}_{u}\left(\mathcal{L}_{v}(\tau)\right)$.

By using the theorem in Section 3.1 and by setting $\mathcal{A}=\mathcal{T}$ where $\mathcal{T}$ is the commutative algebra of tensorfieds, we obtain a deformed tensor algebra $\mathcal{T}_{\star}$ with associative $\star$-tensor product

$$
\tau \otimes_{\star} \tau^{\prime}:=\overline{\mathrm{f}}^{\alpha}(\tau) \otimes \overline{\mathrm{f}}_{\alpha}\left(\tau^{\prime}\right)
$$

It follows that in $\mathcal{T}_{\star}$ we have in particular

$$
\begin{aligned}
\tau \otimes_{\star} h \star \tau^{\prime} & =\tau \star h \otimes_{\star} \tau^{\prime}, \\
h \star\left(\tau \otimes_{\star} \tau^{\prime}\right) & =(h \star \tau) \otimes_{\star} \tau^{\prime} .
\end{aligned}
$$

The $\star$-product between a function and a tensor is noncommutative

$$
\tau \star h=\mathcal{L}_{\overline{\mathrm{f}}^{\alpha}}(\tau) \mathcal{L}_{\overline{\mathrm{f}}_{\alpha}}(h)=\mathcal{L}_{\overline{\mathrm{f}}_{\alpha}}(h) \mathcal{L}_{\overline{\mathrm{f}}^{\alpha}}(\tau)=\mathcal{L}_{\bar{R}^{\alpha}}(h) \star \mathcal{L}_{\bar{R}_{\alpha}}(\tau)=\bar{R}^{\alpha}(h) \star \bar{R}_{\alpha}(\tau) .
$$

We now consider the construction performed at the beginning of this section, but with $\mathcal{T}_{\star}$ instead of $A_{\star}\left(\right.$ or $\left.U \Xi_{\star}\right)$ and obtain that $\mathcal{T}_{\star}$ is a $U \Xi_{\star}$-module algebra. The action of $U \Xi_{\star}$ on $\mathcal{T}_{\star}$ is given by the $\star$-Lie derivative

$$
\mathcal{L}_{\xi}^{\star}(\tau):=\mathcal{L}_{D \xi}(\tau)=\overline{\mathrm{f}}^{\alpha}(\xi)\left(\overline{\mathrm{f}}_{\alpha}(\tau)\right) .
$$

Compatibility with the $\star$-product in $\mathcal{T}_{\star}$ is proven as in $(4.5)$

$$
\mathcal{L}_{\xi}^{\star}\left(\tau \star \tau^{\prime}\right)=\mathcal{L}_{\xi_{1_{\star}}}^{\star}(\tau) \star \mathcal{L}_{\xi_{2_{\star}}}^{\star}\left(\tau^{\prime}\right)
$$

In particular the $\star$-Lie derivative along vectorfields satisfies the deformed Leibniz rule

$$
\mathcal{L}_{u}^{\star}(h \star g)=\mathcal{L}_{u}^{\star}(h) \star g+\bar{R}^{\alpha}(h) \star \mathcal{L}_{\bar{R}_{\alpha}(u)}^{\star}(g) .
$$

in accordance with the coproduct formula (3.12).

\footnotetext{
${ }^{7}$ We assume for simplicity that $\Omega \otimes \ldots \Omega \otimes \Xi \otimes \ldots \Xi \cong \Gamma\left(T^{*} M \otimes \ldots T M \otimes T M \otimes \ldots T M\right)$. That this is always the case for a smooth manifold $M$ (see for example [56], Prop. 2.6.) follows from the existence of a finite covering of $M$ that trivializes the tangent bundle $T M$ and the cotangent bundle $T^{*} M$, see for example [57], Thm. 7.5.16.
} 


\subsubsection{Vectorfields $\Xi_{\star}$ are an $A_{\star}$-bimodule}

From the definition of the product of tensorfields (4.8), considering functions and vectorfields as particular tensors, we see that we can $\star$-multiply functions with vectorfields from the left and from the right. Because of associativity of the tensorprduct we see that the space of vectorfields $\Xi_{\star}$ is an $A_{\star}$-bimodule. In the commutative case left and right action of functions on vectorfields coincide, $u h=h u^{8}$. In the noncommutative case the left and right $A_{\star}$-actions on $\Xi_{\star}$ are not the same, but are related as in (4.11).

\section{Local coordinates description of vectorfields}

In a coordinate neighborhood $U$ with coodinates $x^{\mu}$ any vectorfield $v$ can be expressed in the $\partial_{\mu}$ basis as $v=v^{\mu} \partial_{\mu}$. We have a similar situation in the noncommutative case.

Lemma 1 In a coordinate neighborhood $U$ with coordinates $x^{\mu}$ every vectorfield $v$ can be uniquely written as

$$
v=v_{\star}^{\mu} \star \partial_{\mu} .
$$

where $v_{\star}^{\mu}$ are functions on $U$.

Proof. We know that $v$ can be uniquely written as $v=v^{\mu} \partial_{\mu}$. In order to prove decomposition (4.14) we show that the equation

$$
v_{\star}^{\mu} \star \partial_{\mu}=v^{\mu} \partial_{\mu}
$$

uniquely determines order by order in $\lambda$ the coefficients $v_{\star}^{\mu}$ in terms of the $v^{\mu}$ ones. First we expand $v^{\mu}$, $v_{\star}^{\mu}$ and $\mathcal{F}^{-1}$,

$$
\begin{aligned}
& v^{\mu}=v_{0}^{\mu}+\lambda v_{1}^{\mu}+\lambda^{2} v_{2}^{\mu}+\ldots \quad, \quad v_{\star}^{\mu}=v_{\star 0}^{\mu}+\lambda v_{\star 1}^{\mu}+\lambda^{2} v_{\star 2}^{\mu}+\ldots \\
& \mathcal{F}^{-1}=\overline{\mathrm{f}}^{\alpha} \otimes \overline{\mathrm{f}}_{\alpha}=1 \otimes 1+\lambda \overline{\mathrm{f}}^{\alpha_{1}} \otimes \overline{\mathrm{f}}_{\alpha_{1}}+\lambda^{2} \overline{\mathrm{f}}^{\alpha_{2}} \otimes \overline{\mathrm{f}}_{\alpha_{2}}+\ldots
\end{aligned}
$$

Then from (4.15) we have

$$
v_{\star 0}^{\mu}=v_{0}^{\mu} \quad, \quad v_{\star 1}^{\mu}=v_{1}^{\mu}-\overline{\mathrm{f}}^{\alpha_{1}}\left(v^{\rho}\right) \overline{\mathrm{f}}_{\alpha_{1} \rho}^{\mu}
$$

where $\overline{\mathrm{f}}_{\alpha_{1} \rho}^{\mu} \partial_{\mu}=\overline{\mathrm{f}}_{\alpha_{1}}\left(\partial_{\rho}\right)$. More in general at order $\lambda^{i}$ we have the equation $v_{\star i}^{\mu} \partial_{\mu}+$ $\sum_{j=1}^{i} \overline{\mathrm{f}}^{\alpha_{j}}\left(v_{\star i-j}^{\rho}\right) \overline{\mathrm{f}}_{\alpha_{j} \rho}^{\mu} \partial_{\rho}=v_{i}^{\mu} \partial_{\mu}$ that uniquely determines $v_{\star i}$ in terms of $\mathcal{F}, v^{\mu}$ and $v_{\star j}^{\mu}$ with $j<i$.

Notice that this proof remains true if the local frame $\left\{\partial_{\mu}\right\}$ is replaced by a more general (not necessarily holonomic or $\lambda$ independent) frame $\left\{e_{a}\right\}$. (Hint: $e_{a}=e_{a}^{\mu} \star \partial_{\mu}$, $\left.\partial_{\mu}=e_{\mu}^{a} \star e_{a}\right)$.

\footnotetext{
${ }^{8}$ Here $u h$ is just the vectorfield that on a function $g$ gives $(u h)(g):=u(g) h$. This notation should not be confused with the operator notation $u \circ h=u(h)+h u$.
} 
Along these lines one can define a change of reference frame,

$$
\partial_{\mu} \rightarrow \partial_{\mu}^{\prime}=L_{\mu}^{\nu} \partial_{\nu}=L_{\star \mu}^{\nu} \star \partial_{\nu}
$$

This is a starting point in order to construct noncommutative transition functions for the tangent bundle $T M$.

\subsubsection{1-Forms $\Omega_{\star}$}

From the tensorfield product definition (4.8), we see that the space of 1 -forms is an $A_{\star}$-bimodule. The $A_{\star}$-bimodule structure explicitly reads, $\forall h \in A_{\star}, \omega \in \Omega_{\star}$,

$$
\omega \star h=\mathcal{L}_{\bar{R}_{\star}^{\alpha}}^{\star}(h) \star \mathcal{L}_{\bar{R}_{\star \alpha}}^{\star}(\omega)=\bar{R}^{\alpha}(h) \star \bar{R}_{\alpha}(\omega) .
$$

The action of $U \Xi_{\star}$ on $\Omega_{\star}$ is given in (4.12).

\section{Local coordinates description of 1 -forms and of tensorfields}

As in the case of vectorfields we have that in a coordinate neighborhood $U$ with coordinates $x^{\mu}$ every 1 -form $\omega$ can be uniquely written as

$$
\omega=\omega_{\mu}^{\star} \star d x^{\mu}
$$

with $\omega_{\mu}^{\star}$ functions on $U$, and where $\left\{d x^{\mu}\right\}$ is the usual dual frame of the vectorfields frame $\left\{\partial_{\mu}\right\}$. We can now show

Lemma 2 In a coordinate neighborhood $U$ with coordinates $x^{\mu}$ every tensorfield $\tau^{p, q}$ can be uniquely written as

$$
\tau^{p, q}=\tau_{\star}^{\nu_{1} \ldots \nu_{q}} \ldots \mu_{p} \star d x^{\mu_{1}} \otimes_{\star} \ldots d x^{\mu_{p}} \otimes_{\star} \partial_{\nu_{1}} \otimes_{\star} \ldots \partial_{\nu_{q}}
$$

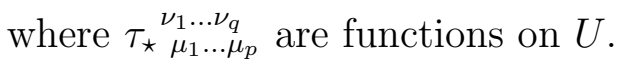

Proof. Following the proof of Lemma 1 we have that $\tau^{p, q}$ can be uniquely written as $\tau^{p, q}=\tau_{\star}^{p, q-1 \nu} \otimes_{\star} \partial_{\nu}$, where $\tau_{\star}^{p, q-1 \nu}$ is a type $(p, q-1)$ tensor. This expression holds for any value of $q$ and therefore (using associativity of the $\otimes_{\star}$ product), $\tau^{p, q}$ can be uniquely written as $\tau^{p, q}=\tau_{\star}^{p, 0 \nu_{1} \nu_{2} \ldots \nu_{q}} \otimes_{\star} \partial_{\nu_{1}} \otimes_{\star} \ldots \partial_{\nu_{q}}$. Similarly, like in formula (4.21), we have

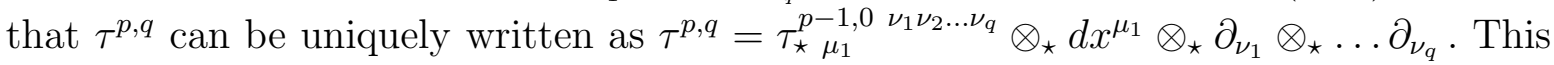
expression holds for any value of $p$ and $q$ and therefore (using associativity of the $\otimes_{\star}$ product) we obtain expression (4.22) and its uniqueness. 


\subsubsection{Exterior algebra of forms $\Omega_{\star}^{\cdot}=\oplus_{p} \Omega_{\star}^{p}$}

As another application of the theorem in Section 3.1 we consider the algebra of exterior forms $\Omega^{*}=\oplus_{p} \Omega^{p}$, and $\star$-deform the wedge product into the $\star$-wedge product,

$$
\vartheta \wedge_{\star} \vartheta^{\prime}:=\overline{\mathrm{f}}^{\alpha}(\vartheta) \wedge \overline{\mathrm{f}}_{\alpha}\left(\vartheta^{\prime}\right)
$$

We denote by $\Omega_{\star}^{\bullet}$ the linear space of forms equipped with the wedge product $\wedge_{\star}$,

$$
\Omega_{\star}^{\bullet}:=\left(\Omega^{\bullet}, \wedge_{\star}\right) \text {. }
$$

As in the commutative case it can be shown [44] that the linear space of exterior forms can be seen as the tensor subspace of totally $\star$-antisymmetric (contravariant) tensorfields. The properties of the $\star$-antisymmetrizator imply that there is a top form that has the same degree as in the undeformed case. This is in accordance with (4.23). Explicitly the $\star$-antisymmetric 2 -form $\omega \wedge_{\star} \omega^{\prime}$ is defined by (cf. (7.1)),

$$
\omega \wedge_{\star} \omega^{\prime}:=\omega \otimes_{\star} \omega^{\prime}-\mathcal{L}_{\overline{R_{\star}}}^{\star}\left(\omega^{\prime}\right) \otimes_{\star} \mathcal{L}_{\bar{R}_{\star \alpha}}^{\star}(\omega) .
$$

It can also be shown [38] that the usual exterior derivative $d: A \rightarrow \Omega$ satisfies the Leibniz rule $d(h \star g)=d h \star g+h \star d g$ and is therefore also the $\star$-exterior derivative. This is so because the exterior derivative commutes with the Lie derivative. In the case that $A$ is a Hopf algebra, the fact that the exterior differential on $A_{\star}$ is not deformed was shown in [54].

\section{$4.2 \star$-Pairing between 1-forms and vectorfields}

Following the general prescription outlined in Section 2.2, we define the $\star$-pairing between vectorfields and 1 -forms as $\langle,\rangle_{\star}:=\langle,\rangle \circ \mathcal{F}^{-1}$. Explicitly, for all $\xi \in \Xi_{\star}, \omega \in \Omega_{\star}$,

$$
\begin{aligned}
\langle,\rangle_{\star}: \Xi_{\star} \otimes_{\mathbb{C}} \Omega_{\star} & \rightarrow A, \\
(\xi, \omega) & \mapsto\langle\xi, \omega\rangle_{\star}:=\left\langle\overline{\mathrm{f}}^{\alpha}(\xi), \overline{\mathrm{f}}_{\alpha}(\omega)\right\rangle .
\end{aligned}
$$

We leave it to the reader to prove the following

Lemma 3 The pairing $\langle,\rangle_{\star}$ is compatible with the $\star$-Lie derivative,

$$
\mathcal{L}_{\xi}^{\star}\left(\langle u, \omega\rangle_{\star}\right)=\left\langle\mathcal{L}_{\xi_{1_{\star}}}^{\star}(u), \mathcal{L}_{\xi_{2_{\star}}}^{\star}(\omega)\right\rangle_{\star},
$$

and satisfies the $A_{\star}$-linearity properties

$$
\begin{gathered}
\langle h \star u, \omega \star k\rangle_{\star}=h \star\langle u, \omega\rangle_{\star} \star k \\
\langle u, h \star \omega\rangle_{\star}=\langle u \star h, \omega\rangle_{\star}=\mathcal{L}_{\bar{R}_{\star}^{\alpha}}^{\alpha}(h) \star\left\langle\mathcal{L}_{\bar{R}_{\star \alpha}}(u), \omega\right\rangle_{\star} .
\end{gathered}
$$


so that $\langle,\rangle_{\star}: \Xi_{\star} \otimes_{\star} \Omega_{\star} \rightarrow A$

In the commutative case we can consider locally a moving frame (or vielbein) $\left\{e_{i}\right\}$ and a dual frame of 1 -forms $\omega^{j}$ :

$$
\left\langle e_{i}, \omega^{j}\right\rangle=\delta_{i}^{j}
$$

in particular $\left\langle\partial_{\mu}, d x^{\nu}\right\rangle=\delta_{\mu}^{\nu}$. In the noncommutative case locally we also have a moving frame $\left\{\hat{e}_{i}\right\}$ and a dual frame of 1 -forms $\omega^{j}$ :

$$
\left\langle\hat{e}_{i}, \omega^{j}\right\rangle_{\star}=\delta_{i}^{j}
$$

We construct it in the following way: since $\left\langle e_{i}, \omega^{j}\right\rangle=\delta_{i}^{j}$ we have $\left\langle e_{i}, \omega^{j}\right\rangle_{\star}=N_{i}^{j}$ with $N$ being a $\star$-invertible matrix since $N_{i}^{j}=\delta_{i}^{j}+O(\lambda)$. We denote by $N^{-1_{\star}}$ the $\star$-inverse matrix of the matrix $N$. We have $N^{-1_{\star}}=1+\lambda N_{1}+\lambda^{2} N_{2}+\ldots$ with the generic terms $N_{n}^{-1_{\star}}$ recursively given by $N_{n}^{-1_{\star}}=-\sum_{l=1}^{n} N_{n-l}^{-1_{\star}} \star N_{l}$, see also [14] for another equivalent explicit expression. Then

$$
\hat{e}_{i}=N_{i}^{-1_{\star} k} \star e_{k}
$$

satisfies $\left\langle\hat{e}_{i}, \omega^{j}\right\rangle_{\star}=\delta_{i}^{j}$ as is easily seen using $A_{\star}$-linearity of the pairing $\langle,\rangle_{\star}$. Of course we also have $\left\langle e_{i}, \hat{\omega}^{j}\right\rangle_{\star}=\delta_{i}^{j}$ with $\hat{\omega}^{j}=\omega^{k} \star N_{k}^{-1_{\star} j}$. We denote by $\left\{\hat{\partial}_{\mu}\right\}$ the basis of vectorfields that satisfy

$$
\left\langle\hat{\partial}_{\mu}, d x^{\nu}\right\rangle_{\star}=\delta_{\mu}^{\nu}
$$

we have $\hat{\partial}_{\mu}=N_{\mu}^{-1_{\star} \nu} \star \partial_{\nu}$ with $N_{\mu}^{\nu}=\left\langle\partial_{\mu}, d x^{\nu}\right\rangle_{\star}$.

Using the pairing $\langle,\rangle_{\star}$ we associate to any 1 -form $\omega$ the left $A_{\star}$-linear map $\langle, \omega\rangle_{\star}$. It can be shown [44] that also the converse holds: any left $A_{\star}$-linear map $\Phi: \Xi_{\star} \rightarrow A_{\star}$ is of the form $\langle, \omega\rangle_{\star}$ for some $\omega$.

\section{Covariant Derivative}

By now we have acquired enough knowledge on $\star$-noncommutative differential geometry to develop the formalism of covariant derivative, torsion, curvature and Ricci tensors just by following the usual classical formalism.

We define a $\star$-covariant derivative $\nabla_{u}^{\star}$ along the vector field $u \in \Xi$ to be a linear map $\nabla_{u}^{\star}: \Xi_{\star} \rightarrow \Xi_{\star}$ such that for all $u, v, z \in \Xi_{\star}, h \in A_{\star}$ :

$$
\begin{aligned}
& \nabla_{u+v}^{\star} z=\nabla_{u}^{\star} z+\nabla_{v}^{\star} z, \\
& \nabla_{h \star u}^{\star} v=h \star \nabla_{u}^{\star} v, \\
& \nabla_{u}^{\star}(h \star v)=\mathcal{L}_{u}^{\star}(h) \star v+\bar{R}^{\alpha}(h) \star \nabla_{\bar{R}_{\alpha}(u)} v
\end{aligned}
$$


Notice that in the last line we have used the coproduct formula $(3.12), \Delta_{\star}(u)=u \otimes 1+$ $\bar{R}_{\star}^{\alpha} \otimes \mathcal{L}_{\bar{R}_{\star \alpha}}^{\star}(u)$. Epression (5.3) is well defined because $\bar{R}_{\alpha}(u)$ is again a vectorfield.

\section{Local coordinates description}

In a coordinate neighborhood $U$ with coordinates $x^{\mu}$ we have the frame $\left\{\hat{\partial}_{\mu}\right\}$ that is «-dual to the frame $\left\{d x^{\mu}\right\}$ (cf. (4.34)). The (noncommutative) connection coefficients $\Gamma_{\mu \nu}^{\sigma}$ are uniquely determined by

$$
\nabla_{\hat{\partial}_{\mu}}^{\star} \hat{\partial}_{\nu}=\Gamma_{\mu \nu}^{\sigma} \star \hat{\partial}_{\sigma}
$$

They uniquely determine the connection, indeed for vectorfields $z$ and $u$ we have,

$$
\begin{aligned}
\nabla_{z}^{\star} u & =\nabla_{z}^{\star}\left(u_{\star}^{\nu} \star \hat{\partial}_{\nu}\right) \\
& =\mathcal{L}_{z}^{\star}\left(u_{\star}^{\nu}\right) \star \hat{\partial}_{\nu}+\bar{R}^{\alpha}\left(u_{\star}^{\nu}\right) \star \nabla \bar{R}_{\alpha}(z) \hat{\partial}_{\nu} \\
& =\mathcal{L}_{z}^{\star}\left(u_{\star}^{\nu}\right) \star \hat{\partial}_{\nu}+\bar{R}^{\alpha}\left(u_{\star}^{\nu}\right) \star \bar{R}_{\alpha}(z)^{\mu} \star \nabla_{\hat{\partial}_{\mu}}^{\star} \hat{\partial}_{\nu} \\
& =\mathcal{L}_{z}^{\star}\left(u_{\star}^{\nu}\right) \star \hat{\partial}_{\nu}+\bar{R}^{\alpha}\left(u_{\star}^{\nu}\right) \star \bar{R}_{\alpha}(z)^{\mu} \star \Gamma_{\mu \nu}{ }^{\sigma} \star \hat{\partial}_{\sigma}
\end{aligned}
$$

where $\bar{R}_{\alpha}(z)^{\mu}$ are the coefficients of $\bar{R}_{\alpha}(z), \bar{R}_{\alpha}(z)=\bar{R}_{\alpha}(z)^{\mu} \star \hat{\partial}_{\mu}$. With respect to a local frame of vectorfields $\left\{e_{i}\right\}$ we have the connection coefficients

$$
\nabla_{e_{i}}^{\star} e_{j}=\Gamma_{i j}^{k} \star e_{k}
$$

\section{Covariant derivative on tensorfields}

We define the covariant derivative on bivectorfields extending by linearity the following deformed Leibniz rule, for all $u, v, z \in \Xi_{\star}$,

$$
\nabla_{u}^{\star}\left(v \otimes_{\star} z\right):=\nabla_{u}^{\star}(v) \otimes_{\star} z+\bar{R}^{\alpha}(v) \otimes_{\star} \nabla_{\bar{R}_{\alpha}(u)} z
$$

We now define the covariant derivative on functions to be the $\star$-Lie derivative,

$$
\nabla_{u}^{\star}(h)=\mathcal{L}_{u}^{\star}(h)
$$

As in the commutative case we also define the covariant derivative on 1 -forms $\Omega_{\star}$, by requiring compatibility with the contraction operator, for all $u, v \in \Xi_{\star}, \omega \in \Omega_{\star}$,

$$
\nabla_{u}^{\star}\langle v, \omega\rangle_{\star}=\left\langle\nabla_{u}^{\star}(v), \omega\right\rangle_{\star}+\left\langle\bar{R}^{\alpha}(v), \nabla_{\bar{R}_{\alpha}(u)}^{\star} \omega\right\rangle_{\star}
$$

so that $\left\langle v, \nabla_{u}^{\star} \omega\right\rangle_{\star}=\mathcal{L}_{\bar{R}^{\alpha}(u)}^{\star}\left\langle\bar{R}_{\alpha}(v), \omega\right\rangle_{\star}-\left\langle\nabla_{\bar{R}^{\alpha}(u)}\left(\bar{R}_{\alpha}(v)\right), \omega\right\rangle_{\star}$. Finally we extend the covariant derivative to all tensorfields via the deformed Leibniz rule (5.7) where now $\tau, \tau^{\prime} \in \mathcal{T}_{\star}$,

$$
\nabla_{u}^{\star}\left(\tau \otimes_{\star} \tau^{\prime}\right):=\nabla_{u}^{\star}(\tau) \otimes_{\star} \tau^{\prime}+\bar{R}^{\alpha}(\tau) \otimes_{\star} \nabla_{\bar{R}_{\alpha}(u)} \tau^{\prime}
$$




\section{Torsion and Curvature}

Definition 2. The torsion $\mathrm{T}$ and the curvature $\mathrm{R}$ associated to a connection $\nabla^{\star}$ are the $\mathbb{C}$-linear maps $\mathrm{T}: \Xi_{\star} \otimes_{\mathbb{C}} \Xi_{\star} \rightarrow \Xi_{\star}$, and $\mathrm{R}^{\star}: \Xi_{\star} \otimes_{\mathbb{C}} \Xi_{\star} \otimes_{\mathbb{C}} \Xi_{\star} \rightarrow \Xi_{\star}$ defined by

$$
\begin{aligned}
\mathrm{T}(u, v) & :=\nabla_{u}^{\star} v-\nabla_{\bar{R}^{\alpha}(v)}^{\star} \bar{R}_{\alpha}(u)-[u, v]_{\star}, \\
\mathrm{R}(u, v, z) & :=\nabla_{u}^{\star} \nabla_{v}^{\star} z-\nabla_{\bar{R}^{\alpha}(v)} \nabla_{\bar{R}_{\alpha}(u)} z-\nabla_{[u v]_{\star}}^{\star} z,
\end{aligned}
$$

for all $u, v, z \in \Xi_{\star}$.

From the antisymmetry property of the bracket []$_{\star}$, see (3.9), and triangularity of the $\mathcal{R}$-matrix it easily follows that the torsion $\mathrm{T}$ and the curvature $\mathrm{R}$ have the following $\star$-antisymmetry property

$$
\begin{aligned}
\mathrm{T}(u, v) & =-\mathrm{T}\left(\bar{R}^{\alpha}(v), \bar{R}_{\alpha}(u)\right), \\
\mathrm{R}(u, v, z) & =-\mathrm{R}\left(\bar{R}^{\alpha}(v), \bar{R}_{\alpha}(u), z\right) .
\end{aligned}
$$

It can be shown [44] that $\mathrm{T}$ and $\mathrm{R}$ are left $A_{\star}$-linear maps,

$$
\begin{aligned}
& \mathrm{T}: \Xi_{\star} \otimes_{\star} \Xi_{\star} \rightarrow \Xi_{\star} \\
& \mathrm{R}: \Xi_{\star} \otimes_{\star} \Xi_{\star} \otimes_{\star} \Xi_{\star} \rightarrow \Xi_{\star}
\end{aligned}
$$

and therefore that they uniquely define a torsion tensor and a curvature tensor. For the torsion, left $A_{\star}$-linearity explicitly reads

$$
\begin{aligned}
& \mathrm{T}(f \star u, v)=f \star \mathrm{T}(u, v), \\
& \mathrm{T}(u, f \star v)=\mathrm{T}(u \star f, v)=\bar{R}^{\alpha}(f) \star \mathrm{T}\left(\bar{R}_{\alpha}(u), v\right),
\end{aligned}
$$

and similarly for the curvature. Instead of entering the technical Hopf algebra aspects of the proof of (6.6) and (6.7), we here present an easy intuitive argument. Recall that $f \star g=\bar{R}^{\alpha}(g) \star \bar{R}_{\alpha}(f)$. In other terms the noncommutativity of the $\star$-product is regulated by the $\mathcal{R}$-matrix. Expression $\bar{R}^{\alpha}(g) \star \bar{R}_{\alpha}(f)$ can be read as saying that the initial ordering $f \star g$ has been inverted. Similarly expression $\bar{R}^{\beta} \bar{R}^{\alpha}(h) \star \bar{R}^{\beta}(f) \star \bar{R}_{\alpha}(g)$ equals $f \star g \star h$ as is easily seen by accounting for the number of elementary transpositions needed to permute $(f, g, h)$ into $(h, f, g)$. In short, $\mathcal{R}^{-1}=\bar{R}^{\alpha} \otimes \bar{R}_{\alpha}$ is a representation of the permutation group on the $\star$-algebra of functions $A_{\star}$, and similarly on the algebra of vectorfields $U \Xi_{\star}$. The formula

$$
[f \star u, v]_{\star}=f \star[u, v]_{\star}-\left(\mathcal{L}_{\bar{R}^{\beta}\left(\bar{R}^{\alpha}(v)\right)} \bar{R}_{\beta}(f)\right) \star \bar{R}_{\alpha}(u)
$$

can then be intuitively obtained recalling the analogue commutative formula $[f u, v]=$ $f[u, v]-\left(\mathcal{L}_{v} f\right) u$ and keeping track of the transpositions occurred. For example the $\mathcal{R}$ matrices in the last addend agree with the reordering $(f, u, v) \rightarrow(v, f, u)$. Recalling again that the inital ordering is $(f, u, v)$ one similarly has

$$
\nabla_{\bar{R}^{\alpha}(v)}^{\star} \bar{R}_{\alpha}(f \star u)=f \star \nabla_{\bar{R}^{\alpha}(v)} \bar{R}_{\alpha}(u)+\left(\mathcal{L}_{\bar{R}^{\beta} \bar{R}^{\alpha}(v)} \bar{R}_{\beta}(f)\right) \star \bar{R}_{\alpha}(u) .
$$


The sum of (6.8) and of (6.9) gives the left $A_{\star}$-linearity property (6.6) of the torsion. Formula (6.7) can be similarly obtained. It also follows from the $\star$-antisymmetry property (6.3).

\section{Local coordinates description}

We denote by $\left\{e_{i}\right\}$ a local frame of vectorfields (subordinate to an open $U \in M$ ) and by $\left\{\theta_{j}\right\}$ the dual frame of 1-forms:

$$
\left\langle e_{i}, \theta^{j}\right\rangle_{\star}=\delta_{i}^{j}
$$

The coefficients $\mathrm{T}_{i j}{ }^{l}$ and $\mathrm{R}_{i j k}{ }^{l}$ of the torsion and curvature tensors with respect to this local frame are defined by

$$
\begin{aligned}
& \mathrm{T}_{i j}{ }^{l}=\left\langle\mathrm{T}\left(e_{i}, e_{j}\right), \theta^{l}\right\rangle_{\star}, \\
& \mathrm{R}_{i j k}{ }^{l}=\left\langle\mathrm{R}\left(e_{i}, e_{j}, e_{k}\right), \theta^{l}\right\rangle_{\star} .
\end{aligned}
$$

We denote by $\Lambda^{\star}$ the $\star$-transposition operator; it is the linear operator given by

$$
\Lambda^{\star}\left(u \otimes_{\star} v\right):=\mathcal{L}_{\bar{R}_{\star}^{\alpha}}^{\star}(v) \otimes_{\star} \mathcal{L}_{\bar{R}_{\star \alpha}}^{\star}(u)=\bar{R}^{\alpha}(v) \otimes_{\star} \bar{R}_{\alpha}(u) .
$$

It is easily seen to be compatible with the $A_{\star}$-bimodule and the $U \Xi_{\star}$-module structure of $\Xi_{\star} \otimes_{\star} \Xi_{\star}$ :

$$
\begin{aligned}
\Lambda^{\star}\left(h \star u \otimes_{\star} v \star k\right) & =h \star \Lambda^{\star}\left(u \otimes_{\star} v\right) \star k, \\
\mathcal{L}_{\xi}^{\star}\left(\Lambda^{\star}\left(u \otimes_{\star} v\right)\right) & =\Lambda^{\star}\left(\mathcal{L}_{\xi}^{\star}\left(u \otimes_{\star} v\right)\right) .
\end{aligned}
$$

Hint: use $(2.51),(2.52)$, and (3.20). Because of the $A_{\star}$-bilinearity property $(6.12)$, we have that $\Lambda^{\star}$ is completely determined by its action on a basis of vectorfields. We define the coefficients $\Lambda_{i j}^{\star k l}$ of $\Lambda^{\star}$ by the expression

$$
\Lambda^{\star}\left(e_{i} \otimes_{\star} e_{j}\right)=\Lambda_{i j}^{\star k l} \star e_{k} \otimes_{\star} e_{l}
$$

Recalling the $\star$-antisymmetry property of $\mathrm{T}$ and $\mathrm{R}$, (see (6.3) and (6.4)), we then immediately have the $\star$-antisymmetry properties of the coefficients $\mathrm{T}_{i j}{ }^{l}$ and $\mathrm{R}_{n i j}{ }^{l}$,

$$
\mathrm{T}_{i j}^{l}=-\Lambda_{i j}^{\star k m} \star \mathrm{T}_{k m}^{l}, \quad \mathrm{R}_{n i j}^{l}=-\Lambda_{i j}^{\star k m} \star \mathrm{R}_{n k m}{ }^{l}
$$

In the commutative case, if the connection is chosen to have vanishing torsion, we have the first Bianchi identities $\mathrm{R}_{i j k}^{l}+\mathrm{R}_{j k i}^{l}+\mathrm{R}_{k i j}^{l}=0$, where the lower indices $i j k$ have been cyclically permuted. There is a similar equation in the noncommutative case. 
We first define the $\star$-operation of cyclic permutaion of three vectors. Recalling the definition of the $\star$-transposition operator we have that $\star$-cyclic permutation of the vectors $u v z$ is given by,

$$
\mathcal{C}^{\star}\left(u \otimes_{\star} v \otimes_{\star} z\right)=u \otimes_{\star} v \otimes_{\star} z+\Lambda_{12}^{\star} \Lambda_{23}^{\star}\left(u \otimes_{\star} v \otimes_{\star} z\right)+\Lambda_{23}^{\star} \Lambda_{12}^{\star}\left(u \otimes_{\star} v \otimes_{\star} z\right)
$$

where $\Lambda_{12}^{\star}=\Lambda \otimes_{\star} i d$ and $\Lambda_{23}^{\star}=i d \otimes_{\star} \Lambda$. From the $A_{\star}$-bilinearity property of $\Lambda^{\star}$ we see that also $\mathcal{C}^{\star}$ is $A_{\star}$-bilinear

$$
\mathcal{C}^{\star}\left(h \star u \otimes_{\star} v \otimes_{\star} z \star k\right)=h \star \mathcal{C}^{\star}\left(u \otimes_{\star} v \otimes_{\star} z\right) \star k
$$

Since any tensor in $\Xi_{\star} \otimes_{\star} \Xi_{\star} \otimes_{\star} \Xi_{\star}$ is of the form $f^{i j k} \star e_{i} \otimes_{\star} e_{j} \otimes_{\star} e_{k}$, we have that the $\star-$ cyclic permutation operator is completely defined by its action on a basis $\left\{e_{i} \otimes_{\star} e_{j} \otimes_{\star} e_{k}\right\}$. This action is completely determined by the coefficients $\mathcal{C}^{\star}{ }_{i j k} n$ of $\mathcal{C}^{\star}$,

$$
\mathcal{C}^{\star}\left(e_{i} \otimes_{\star} e_{j} \otimes_{\star} e_{k}\right)=\mathcal{C}_{i j k}^{\star l m n} \star e_{l} \otimes_{\star} e_{m} \otimes_{\star} e_{n}
$$

We can now state the first Bianchi identity in the case of vanishing torsion:

$$
\mathcal{C}^{\star}(\mathrm{R}(u, v, z))=0
$$

where $\mathcal{C}^{\star}$ denotes $\star$-cyclic permutation of $u, v$, and $z$. In components the Bianchi identity reads

$$
\mathcal{C}^{\star l m k}{ }_{i j k} \star \mathrm{R}_{l m n}^{p}=0
$$

The proof of the Bianchi identity follows the classical proof. Since the torsion vanishes we have $\nabla_{u}^{\star} \mathrm{T}(v, z)=0$, this equation reads

$$
\nabla_{u}^{\star} \nabla_{v}^{\star}(z)-\nabla_{u}^{\star} \nabla_{\bar{R}^{\alpha}(z)} \bar{R}_{\alpha}(v)-\nabla_{\bar{R}^{\alpha}\left([v, z]_{\star}\right)} \bar{R}_{\alpha}(u)-\left[u,[v, z]_{\star}\right]_{\star},
$$

where we have used that $\mathrm{T}\left(u,[v, z]_{\star}\right)=0$. We now add three times this equation, each time $\star$-cyclically permuting the vectors $(u, v, z)$, so that we have the three orderings $(u, v, z),\left(\bar{R}^{\beta} \bar{R}^{\alpha}(z), \bar{R}_{\beta}(u), \bar{R}_{\alpha}(v)\right)$ and $\left(\bar{R}^{\delta}(v), \bar{R}^{\gamma}(z), \bar{R}_{\gamma} \bar{R}_{\delta}(u)\right)$. The three addends

$$
\left[u,[v, z]_{\star}\right]_{\star}+\star \text {-cyclic perm. }
$$

vanish because of the $\star$-Jacoby identities, the remaining addends give the Bianchi identity. (This can be seen using (2.51), (2.52) and the quantum Yang-Baxter equation $\mathcal{R}_{12} \mathcal{R}_{13} \mathcal{R}_{23}=\mathcal{R}_{23} \mathcal{R}_{13} \mathcal{R}_{12}$, that is a consequence of (2.50), (2.51), (2.52)).

We end this section with the definition of the Ricci tensor. In the commutative case the Ricci tensor is a contraction of the curvature tensor, $\operatorname{Ric}_{j k}=\mathrm{R}_{i j k}{ }^{i}$. We define the Ricci map to be the following contraction of the curvature:

$$
\operatorname{Ric}(u, v):=\left\langle\theta^{i}, \mathrm{R}\left(e_{i}, u, v\right)\right\rangle_{\star}^{\prime},
$$


where sum over $i$ is understood. The contraction $\langle,\rangle_{\star}^{\prime}$ is a contraction between forms on the left and vectorfields on the right. It is defined through the by now familiar deformation of the commutative pairing,

$$
\begin{aligned}
\langle\omega, u\rangle_{\star}^{\prime}: & =\left\langle\overline{\mathrm{f}}^{\alpha}(\omega), \overline{\mathrm{f}}_{\alpha}(u)\right\rangle, \\
& =\left\langle\bar{R}^{\alpha}(u), \bar{R}_{\alpha}(\omega)\right\rangle_{\star} .
\end{aligned}
$$

The pairing $\langle,\rangle_{\star}^{\prime}$ has of course the $A_{\star}$-linearity properties

$$
\langle h \star \omega, u \star k\rangle_{\star}^{\prime}=h \star\langle\omega, u\rangle_{\star}^{\prime} \star k, \quad\langle\omega, h \star u\rangle_{\star}^{\prime}=\langle\omega \star h, u\rangle_{\star}^{\prime} .
$$

Definition (6.21) is well given because it is independent from the choice of the frame $\left\{e_{i}\right\}$ (and the dual frame $\left\{\theta^{i}\right\}$ ), and because the Ricci map so defined is an $A_{\star}$-linear map:

$$
\begin{aligned}
& \operatorname{Ric}(f \star u, v)=f \star \operatorname{Ric}(u, v) \\
& \operatorname{Ric}(u, f \star v)=\operatorname{Ric}(u \star f, v)=\bar{R}^{\alpha}(f) \star \operatorname{Ric}\left(\bar{R}_{\alpha}(u), v\right) .
\end{aligned}
$$

In order to prove this statement we consider the coefficients $\mathrm{R}^{j}\left(e_{i}, u, v\right)$ of the vector

$$
\mathrm{R}\left(e_{i}, u, v\right)=\mathrm{R}^{j}\left(e_{i}, u, v\right) \star e_{j} .
$$

$A_{\star}$-linearity of $\mathrm{R}$ implies $A_{\star}$-linearity of the coefficients, $\mathrm{R}^{j}\left(h \star e_{i}, u, v\right)=h \star \mathrm{R}^{j}\left(e_{i}, u, v\right)$. This in turn implies (recall end of Section 4) that there exists 1-forms $\omega_{\mathrm{R}}^{j}(u, v)$ such that

$$
\mathrm{R}^{j}\left(e_{i}, u, v\right)=\left\langle e_{i}, \omega_{\mathrm{R}}^{j}(u, v)\right\rangle_{\star} .
$$

From $\mathrm{R}\left(e_{i}, h \star u, v\right)=\mathrm{R}\left(e_{i} \star h, u, v\right)$ we immediately see that the 1-forms $\omega_{\mathrm{R}}^{j}(u, v)$ are left linear in $u$, i.e., $\omega_{\mathrm{R}}^{j}(h \star u, v)=h \star \omega_{\mathrm{R}}^{j}(u, v)$. We now have

$$
\begin{aligned}
\left\langle\theta^{i}, \mathrm{R}\left(e_{i}, u, v\right)\right\rangle_{\star}^{\prime} & =\left\langle\theta^{i} \star \mathrm{R}^{j}\left(e_{i}, u, v\right), e_{j}\right\rangle_{\star}^{\prime} \\
& =\left\langle\theta^{i} \star\left\langle e_{i}, \omega_{\mathrm{R}}^{j}(u, v)\right\rangle_{\star}, e_{j}\right\rangle_{\star}^{\prime} \\
& =\left\langle\omega_{\mathrm{R}}^{j}(u, v), e_{j}\right\rangle_{\star}^{\prime}
\end{aligned}
$$

where in the first line we used (6.23). This formula implies independence from the choice of basis $\left\{e_{i}\right\}$ and left $A_{\star}$-linearity of Ric.

The coefficients of the Ricci tensor are

$$
\operatorname{Ric}_{j k}=\operatorname{Ric}\left(e_{j}, e_{k}\right) \text {. }
$$




\section{$7 \quad$ Metric and Einstein Equations}

In order to define a $\star$-metric we need to define $\star$-symmetric elements in $\Omega_{\star} \otimes_{\star} \Omega_{\star}$. In (6.11) we have defined the transposition operator $\Lambda^{\star}$ on vectorfields; we can similarly define it on forms,

$$
\Lambda^{\star}\left(\omega \otimes_{\star} \omega^{\prime}\right):=\mathcal{L}_{\bar{R}_{\star}^{\alpha}}^{\star}\left(\omega^{\prime}\right) \otimes_{\star} \mathcal{L}_{\bar{R}_{\star \alpha}}^{\star}(\omega)=\bar{R}^{\alpha}\left(\omega^{\prime}\right) \otimes_{\star} \bar{R}_{\alpha}(\omega) .
$$

We now recall that $\Omega_{\star} \otimes_{\star} \Omega_{\star}=\Omega \otimes \Omega$ as vectorspaces, and we notice that the transposition operator $\Lambda^{\star}: \Omega_{\star} \otimes_{\star} \Omega_{\star} \rightarrow \Omega_{\star} \otimes_{\star} \Omega_{\star}$ is just the classical transposition operator $\Lambda: \Omega \otimes \Omega \rightarrow$ $\Omega \otimes \Omega$. Indeed we have

$$
\Lambda\left(\omega \otimes_{\star} \omega^{\prime}\right)=\Lambda\left(\overline{\mathrm{f}}^{\alpha}(\omega) \otimes \overline{\mathrm{f}}_{\alpha}\left(\omega^{\prime}\right)\right)=\overline{\mathrm{f}}_{\alpha}\left(\omega^{\prime}\right) \otimes \overline{\mathrm{f}}^{\alpha}(\omega)=\bar{R}^{\alpha}\left(\omega^{\prime}\right) \otimes_{\star} \bar{R}_{\alpha}(\omega)=\Lambda^{\star}\left(\omega \otimes_{\star} \omega^{\prime}\right)
$$

where in the first equality we have explicitly written the element $\omega \otimes_{\star} \omega^{\prime}$ as an element of $\Omega \otimes \Omega$, and then in the second equality we have applied the definition of $\Lambda$. This implies that (anti-)symmetric elements in $\Omega \otimes \Omega$ are $\star$-(anti-)symmetric elements in $\Omega_{\star} \otimes_{\star} \Omega_{\star}$.

Since a commutative metric is a nondegenerate symmetric tensor in $\Omega \otimes \Omega$ we conclude that any commutative metric is also a noncommutative metric, ( $\star$-nondegeneracy of the metric is insured by the fact that at zeroth order in the deformation parameter $\lambda$ the metric is nondegenerate). Contrary to [8], [55], we see that in our approach, where all (moving) frames are on equal footing, there are infinitely many metrics compatible with a given noncommutative differential geometry, noncommutativity does not single out a preferred metric.

We denote by $g$ the metric tensor. If we write

$$
\mathrm{g}=\mathrm{g}^{a} \otimes_{\star} \mathrm{g}_{a} \in \Omega_{\star} \otimes_{\star} \Omega_{\star}
$$

(for example locally $\mathrm{g}=\theta^{j} \otimes_{\star} \theta^{i} \star \mathrm{g}_{i j}$ ), then for every $v \in \Xi_{\star}$ we can define the 1 -form

$$
\langle v, \mathrm{~g}\rangle_{\star}:=\left\langle v, \mathrm{~g}^{a}\right\rangle_{\star} \star \mathrm{g}_{a}
$$

and we can then construct the left $A_{\star}$-linear map g, corresponding to the metric tensor $\mathrm{g} \in \Omega_{\star} \otimes_{\star} \Omega_{\star}$, as

$$
\begin{aligned}
\mathrm{g}: \Xi_{\star} \otimes_{\star} \Xi_{\star} & \rightarrow A_{\star} \\
(u, v) & \mapsto \mathrm{g}(u, v)=\left\langle u \otimes_{\star} v, \mathrm{~g}\right\rangle_{\star}:=\left\langle u,\langle v, \mathrm{~g}\rangle_{\star}\right\rangle_{\star} .
\end{aligned}
$$

The $\star$-inverse metric $\mathrm{g}^{-1} \in \Xi_{\star} \otimes_{\star} \Xi_{\star}$ is then defined by the following equations, for all $u \in \Xi_{\star}, \omega \in \Omega_{\star}$,

$$
\begin{aligned}
& \left\langle\langle u, \mathrm{~g}\rangle_{\star}, \mathrm{g}^{-1}\right\rangle_{\star}^{\prime}=u, \\
& \left\langle\left\langle\omega, \mathrm{g}^{-1}\right\rangle_{\star}^{\prime}, \mathrm{g}\right\rangle_{\star}=\omega,
\end{aligned}
$$


where, as in (7.3), we have defined

$$
\left\langle\omega, \mathrm{g}^{-1}\right\rangle_{\star}^{\prime}:=\left\langle\omega, \mathrm{g}^{a-1}\right\rangle_{\star}^{\prime} \star \mathrm{g}_{a}^{-1}
$$

and we have decomposed $\mathrm{g}^{-1}$ as

$$
\mathrm{g}^{-1}=\mathrm{g}^{a-1} \otimes_{\star} \mathrm{g}_{a}^{-1} \in \Xi_{\star} \otimes_{\star} \Xi_{\star}
$$

(for example locally $\mathrm{g}^{-1}=\mathrm{g}^{i j_{\star}} \star e_{j} \otimes_{\star} e_{i}$ ). At zeroth order in the deformation parameter $\lambda$, and using local coordinates, we write $\mathrm{g}=\mathrm{g}_{\mu \nu} d x^{\mu} \otimes d x^{\nu}$ and the above definition of the inverse metric gives $\mathrm{g}^{-1}=\mathrm{g}^{\mu \nu} \partial_{\mu} \otimes \partial_{\nu}$, where $\mathrm{g}^{\mu \nu}$ is the inverse matrix of $\mathrm{g}_{\mu \nu}, \mathrm{g}^{\mu \nu} \mathrm{g}_{\nu \rho}=\delta_{\rho}^{\mu}$, $\mathrm{g}_{\mu \nu} \mathrm{g}^{\nu \rho}=\delta_{\mu}^{\rho}$. For the noncommutative analogue of the relations $\mathrm{g}^{\mu \nu} \mathrm{g}_{\nu \rho}=\delta_{\rho}^{\mu}, \mathrm{g}_{\mu \nu} \mathrm{g}^{\nu \rho}=\delta_{\mu}^{\rho}$ see the end of the next section.

Consider now the connection that has vanishing torsion and that is metric compatible, $\nabla_{u}^{\star} \mathrm{g}=0$. See [44], see also [14] for the case $\theta$-const.. The scalar curvature $\mathfrak{R}$ with respect to this connection is given by

$$
\mathfrak{R}:=\operatorname{Ric}\left(\mathrm{g}^{a-1}, \mathrm{~g}_{a}^{-1}\right)
$$

where $\mathrm{g}^{-1}=\mathrm{g}^{a-1} \otimes_{\star} \mathrm{g}_{a}^{-1} \in \Xi_{\star} \otimes_{\star} \Xi_{\star}$. Locally we have $\mathrm{g}^{-1}=\mathrm{g}^{i j_{\star}} \star e_{j} \otimes_{\star} e_{i}$, and

$$
\begin{aligned}
\mathfrak{R} & =\operatorname{Ric}\left(\mathrm{g}^{i j_{\star}} \star e_{j}, e_{i}\right)=\mathrm{g}^{i j_{\star}} \star \operatorname{Ric}\left(e_{j}, e_{i}\right) \\
& =\mathrm{g}^{i{ }^{\prime}} \star \operatorname{Ric}_{j i} .
\end{aligned}
$$

We finally arrive at the noncommutative Einstein equation (in vacuum),

$$
\text { Ric }-\frac{1}{2} g \star \mathfrak{R}=0
$$

where the dynamical field is the metric $g$. This equation is an equality between the left $A_{\star}$-linear maps Ric and $\mathrm{g} \star \mathfrak{R}$, where

$$
(\mathrm{g} \star \mathfrak{R})(u, v):=\left\langle u \otimes_{\star} v, \mathrm{~g} \star \mathfrak{R}\right\rangle_{\star}=\left\langle u \otimes_{\star} v, \mathrm{~g}\right\rangle_{\star} \star \mathfrak{R}=\mathrm{g}(u, v) \star \mathfrak{R} .
$$

Because of left $A_{\star}$-linearity the curvature scalar must appear on the right of the metric and not on the left in (7.11). Applying (7.11) to the vectors $e_{i}$ and $e_{j}$ we obtain the components equation

$$
\operatorname{Ric}_{i j}-\frac{1}{2} \mathrm{~g}_{i j} \star \Re=0,
$$

where $\mathrm{g}_{i j}=\mathrm{g}\left(e_{i}, e_{j}\right)=\left\langle e_{i} \otimes_{\star} e_{j}, \mathrm{~g}\right\rangle_{\star}$ are the same coefficients appearing in the expression $\mathrm{g}=\theta^{j} \otimes_{\star} \theta^{i} \star \mathrm{g}_{i j}$. 


\section{Conjugation}

In this section we introduce the notion of complex conjugation on the algebra $A_{\star}$, and we see that we can impose reality conditions on the $\star$-spaces of functions, vectorfields and tensorfields.

We first briefly recall the commutative $*$-structure. Given a smooth real manifold $M$, the usual $*$-structure on the complex valued functions $A=\operatorname{Fun}(M)$ is a map $*: A \rightarrow A$, where for all $h \in A$ and $m \in M$,

$$
h^{*}(m)=\overline{h(m)} \text {, }
$$

here the bar - denotes complex conjugation. This $*$-structure induces a $*$-structure on the Lie algebra of vectorfields by defining $*: \Xi \rightarrow \Xi$, where for all $u \in \Xi$ and $h \in A$,

$$
u^{*}(h):=\left(S(u)\left(h^{*}\right)\right)^{*}=-\left(u\left(h^{*}\right)\right)^{*} .
$$

It is easy to check that the $*$-operation so defined is antimultiplicative with respect to the Lie bracket of $\Xi,[u, v]^{*}=\left[v^{*}, u^{*}\right]$. In particular, locally, we can consider the real coordinate functions $x^{\mu}$, then the partial derivatives $\partial_{\mu}$ are pure imaginary, $\partial_{\mu}^{*}=-\partial_{\mu}$; we also have $u^{*}=\left(u^{\mu} \partial_{\mu}\right)^{*}=-\overline{u^{\mu}} \partial_{\mu}$.

The $*$-structure on $\Xi$ is extended to the universal enveloping algebra $U \Xi$ by antilinearity and antimultiplicativity, so that for all $\xi, \zeta \in U \Xi,(\xi \zeta)^{*}=\zeta^{*} \xi^{*}$. Applying a vectorfield $v$ to definition (8.2) we obtain $\left(v^{*} u^{*}\right)(h)=\left(S(u v)\left(h^{*}\right)\right)^{*}$, and iterating we obtain that for a generic element of $U \Xi$,

$$
\xi^{*}(h)=\left(S(\xi)\left(h^{*}\right)\right)^{*} .
$$

Similarly from $u^{*}(v)=\left[u^{*}, v\right]=\left[S(u), v^{*}\right]^{*}=\left(S(u)\left(v^{*}\right)\right)^{*}$ we have,

$$
\xi^{*}(\zeta)=\left(S(\xi)\left(\zeta^{*}\right)\right)^{*}
$$

Finally, from the local formula $\left\langle\partial_{\mu}, d x^{\nu}\right\rangle^{*}=-\left\langle\partial_{\mu}^{*},\left(d x^{\nu}\right)^{*}\right\rangle^{*}$ we have the general formula of compatibility between the $*$-structure and the pairing

$$
\langle u, \omega\rangle^{*}=-\left\langle u^{*}, \omega^{*}\right\rangle \text {. }
$$

We now study the $*$-operation in the noncommutative context. We define the $*$ structure on $A_{\star}$ to be the same as that on $A$. The requirement

$$
(h \star g)^{*}=g^{*} \star h^{*}
$$

is then satisfied if the twist $\mathcal{F}$ satisfies the relation $(S \otimes S) \mathcal{F}_{21}=\mathcal{F}^{* \otimes *}$, i.e.,

$$
(S \otimes S) \mathcal{F}_{21}^{-1}=\mathcal{F}^{-1 * \otimes *} .
$$


We similarly define the $*$-structure on $U \Xi$ to be the same as the undeformed one. Using (8.4) it is not difficult to show that the $*$-operation is compatible with the $\star$ product of $U \Xi_{\star}$ and with the $\star$-Lie bracket of $\Xi_{\star}$,

$$
(\xi \star \zeta)^{*}=\zeta^{*} \star \xi^{*} \quad, \quad[u, v]_{\star}^{*}=\left[v^{*}, u^{*}\right]_{\star} .
$$

It can be shown [44] that the $*$-operation is compatible with the triangular Hopf algebra structure of $U \Xi_{\star}$ (a key point being that on $U \Xi^{\mathcal{F}}$ the $*$-operation reads $\xi^{* \mathcal{F}}:=\chi \xi^{*} \chi^{-1}$ ). On tensors too the $*$-structure is by definition the undeformed one, and we have, for all $\tau, \tau^{\prime} \in \mathcal{T}_{\star}$,

$$
\left(\tau \otimes_{\star} \tau^{\prime}\right)^{*}=\bar{R}^{\alpha}\left(\tau^{*}\right) \otimes_{\star} \bar{R}_{\alpha}\left(\tau^{\prime *}\right) .
$$

Finally the two pairings $\langle,\rangle_{\star}$ and $\langle,\rangle_{\star}^{\prime}$ are related by the $*$-operation, for all $u \in \Xi_{\star}$ and $\omega \in \Omega_{\star}$, we have

$$
\langle u, \omega\rangle_{\star}^{*}=-\left\langle\omega^{*}, u^{*}\right\rangle_{\star}^{\prime}
$$

In particular, if locally we consider a basis $\left\{e_{i}\right\}$ and the dual basis $\left\{\theta^{i}\right\}$,

$$
\left\langle e_{i}, \theta^{j}\right\rangle_{\star}=\delta_{i}^{j}
$$

then the $*$-conjugate basis $\left\{e_{i}^{*}\right\}$ and $\left\{\theta^{j *}\right\}$ are (up to a sign) dual with respect to the $\langle,\rangle_{\star}^{\prime}$ pairing,

$$
\left\langle\theta^{j *}, e_{i}^{*}\right\rangle_{\star}^{\prime}=-\delta_{i}^{j}
$$

We can now study for example the reality property

$$
\mathrm{g}^{*}=\mathrm{g}
$$

of the metric tensor $\mathrm{g} \in \Omega_{\star} \otimes_{\star} \Omega_{\star}$. The metric tensor has a convenient expansion in terms of the $\theta^{i}$ and the $\theta^{\bar{j}} 1$-forms (here $\bar{\jmath}$ is just an index like $i$ or $j$ ). We set

$$
\mathrm{g}=\theta^{i} \otimes_{\star} \mathrm{g}_{i \bar{\jmath}} \star \theta^{\bar{\jmath} *} \text {. }
$$

In this basis reality of the metric, and therefore of the noncommutative Einstein equations, has a very simple explicit expression. Also the explicit expression for the inverse metric is particularly simple in this basis.

We first study the consequences of the reality condition $\mathrm{g}=\mathrm{g}^{*}$ on the metric coefficients $g_{i \bar{\jmath}}$. From (8.9) we have,

$$
\mathrm{g}^{*}=\bar{R}^{\alpha}\left(\theta^{i *}\right) \otimes_{\star} \bar{R}_{\alpha}\left(\theta^{\bar{\jmath}} \star \mathrm{g}_{i \bar{\jmath}}^{*}\right)=\bar{R}^{\alpha}\left(\theta^{\bar{\jmath} *}\right) \otimes_{\star} \bar{R}_{\alpha}\left(\theta^{i} \star \mathrm{g}_{\bar{\jmath} i}^{*}\right)
$$

where in the last equality we have just renamed the indices. In order to compare this expression of $\mathrm{g}^{*}$ with the expression (8.13) of $\mathrm{g}$, we use the $\star$-symmetry property of the metric, $\mathrm{g}=\Lambda^{\star} \mathrm{g}$, to rewrite the metric as

$$
\mathrm{g}=\theta^{i} \star \mathrm{g}_{i \bar{\jmath}} \otimes_{\star} \theta^{\bar{\jmath} *}=\bar{R}^{\alpha}\left(\theta^{\bar{\jmath} *}\right) \otimes_{\star} \bar{R}_{\alpha}\left(\theta^{i} \star \mathrm{g}_{i \bar{\jmath}}\right) .
$$


Comparison with (8.14) gives, $\bar{R}^{\alpha}\left(\theta^{\bar{j} *}\right) \otimes_{\star} \bar{R}_{\alpha}\left(\theta^{i} \star \mathrm{g}_{\bar{\jmath} i}^{*}\right)=\bar{R}^{\alpha}\left(\theta^{\bar{\jmath} *}\right) \otimes_{\star} \bar{R}_{\alpha}\left(\theta^{i} \star \mathrm{g}_{i \bar{\jmath}}\right)$ iff $\mathrm{g}=\mathrm{g}^{*}$. After applying the transposition $\Lambda^{\star}$ to this equation we obtain that reality of $\mathrm{g}$ reads

$$
\theta^{i} \star \mathrm{g}_{\bar{\jmath} i}^{*} \otimes_{\star} \theta^{\bar{\jmath} *}=\theta^{i} \star \mathrm{g}_{i \bar{\jmath}} \otimes_{\star} \theta^{\bar{\jmath} *}
$$

i.e.,

$$
\mathrm{g}_{\bar{\jmath} i}^{*}=\mathrm{g}_{i \bar{\jmath}}
$$

Concerning the inverse metric $\mathrm{g}^{-1}$, we have that it is given by the expression

$$
\mathrm{g}^{-1}=-e_{\bar{\imath}}^{*} \otimes_{\star} \mathrm{g}^{\bar{i} j_{\star}} \star e_{j}
$$

where $\mathrm{g}^{\bar{i} j_{\star}}$ is the $\star$-inverse matrix of $\mathrm{g}_{i \bar{\jmath}}$,

$$
\mathrm{g}^{\bar{i} j_{\star}} \star \mathrm{g}_{j \bar{\ell}}=\delta_{\bar{\ell}}^{\bar{\imath}}, \quad \mathrm{g}_{i \bar{\jmath}} \star \mathrm{g}^{\bar{\jmath} \ell \star}=\delta_{i}^{\ell} .
$$

Indeed it is not difficult to see that (8.16) satisfies (7.5) and (7.6).

\section{Acknowledgements}

We are very thankful to Christian Blohmann and Peter Schupp for many useful discussions and insights. Part of this work is based on our common work [14]. We would also like to thank Gaetano Fiore, John Madore and Stefan Waldmann for their comments. Hospitality and support from Arnold Sommerfeld Center for Theoretical Physics and Max-Planck-Institut für Physik München, and from INFN Torino and Universitá di Torino (MIUR contract PRIN 2003023852) is acknowledged.

\section{A Appendix}

\section{A.1 Proof that $U \Xi^{\mathcal{F}}$ is a Hopf Algebra}

We start from

$$
(\varepsilon \otimes i d) \Delta^{\mathcal{F}}(u)=u=(i d \otimes \varepsilon) \Delta^{\mathcal{F}}(u)
$$

and calculate first the left hand side

$$
\begin{aligned}
(\varepsilon \otimes i d) \Delta^{\mathcal{F}}(u) & =(\varepsilon \otimes i d)\left(\mathrm{f}^{\alpha} u_{1} \overline{\mathrm{f}}^{\beta} \otimes \mathrm{f}_{\alpha} u_{2} \overline{\mathrm{f}}_{\beta}\right) \\
& =\varepsilon\left(\mathrm{f}^{\alpha} u_{1} \overline{\mathrm{f}}^{\beta}\right) \mathrm{f}_{\alpha} u_{2} \overline{\mathrm{f}}_{\beta}=\varepsilon\left(\mathrm{f}^{\alpha}\right) \varepsilon\left(u_{1}\right) \varepsilon\left(\overline{\mathrm{f}}^{\beta}\right) \mathrm{f}_{\alpha} u_{2} \overline{\mathrm{f}}_{\beta}
\end{aligned}
$$

In the last line we have used that $\varepsilon: U \Xi \rightarrow \mathbb{C}$ is an algebra homomorphism. Applying $(\varepsilon \otimes i d)$ on the identity

$$
\mathcal{F F}^{-1}=1 \otimes 1
$$


and using (2.29) gives

$$
\begin{aligned}
1=(\varepsilon \otimes i d) \mathcal{F} \mathcal{F}^{-1} & =(\varepsilon \otimes i d)\left(\mathrm{f}^{\alpha} \overline{\mathrm{f}}^{\beta} \otimes \mathrm{f}_{\alpha} \overline{\mathrm{f}}_{\beta}\right) \\
& =\varepsilon\left(\mathrm{f}^{\alpha}\right) \varepsilon\left(\overline{\mathrm{f}}^{\beta}\right) \mathrm{f}_{\alpha} \overline{\mathrm{f}}_{\beta}=\varepsilon\left(\overline{\mathrm{f}}^{\beta}\right) \overline{\mathrm{f}}_{\beta} .
\end{aligned}
$$

Inserting this into (A.2) we finally obtain

$$
(\varepsilon \otimes i d) \Delta^{\mathcal{F}}(u)=\varepsilon\left(u_{1}\right) u_{2}=u .
$$

In order to calculate the right hand side of (A.1) one proceeds in the analogous way.

Next we prove

$$
\mu\left(S^{\mathcal{F}} \otimes i d\right) \Delta^{\mathcal{F}}(u)=\varepsilon(u) 1=\mu\left(i d \otimes S^{\mathcal{F}}\right) \Delta^{\mathcal{F}}(u)
$$

To show this we first have to prove that $\chi^{-1}=S\left(\overline{\mathrm{f}}^{\alpha}\right) \overline{\mathrm{f}}_{\alpha}$ :

$$
\begin{aligned}
\chi \chi^{-1} & =\mathrm{f}^{\beta} S\left(\mathrm{f}_{\beta}\right) S\left(\overline{\mathrm{f}}^{\alpha}\right) \overline{\mathrm{f}}_{\alpha} \\
& =\overline{\mathrm{f}}^{\gamma} \varepsilon\left(\overline{\mathrm{f}}_{\gamma}\right) \mathrm{f}^{\beta} S\left(\overline{\mathrm{f}}^{\alpha} \mathrm{f}_{\beta}\right) \overline{\mathrm{f}}_{\alpha} \\
& =\overline{\mathrm{f}}^{\gamma} \mathrm{f}^{\beta} S\left(\overline{\mathrm{f}}_{\gamma_{1}} \overline{\mathrm{f}}^{\alpha} \mathrm{f}_{\beta}\right) \overline{\mathrm{f}}_{\gamma_{2}} \overline{\mathrm{f}}_{\alpha} \\
& \left.=\overline{\mathrm{f}}_{1}^{\gamma} S\left(\overline{\mathrm{f}}_{2}^{\gamma}\right)\right) \overline{\mathrm{f}}_{\gamma} \\
& =\varepsilon\left(\overline{\mathrm{f}}^{\gamma}\right) \overline{\mathrm{f}}_{\gamma}=1 .
\end{aligned}
$$

In the first line we used the definitions given in (2.47). Next we inserted $1=\overline{\mathrm{f}}^{\gamma} \varepsilon\left(\overline{\mathrm{f}}_{\gamma}\right)$ which we showed in (A.4). The antipode property $S\left(\xi_{1}\right) \xi_{2}=\varepsilon(\xi)$ together with the fact that the antipode is an antialgebra homomorphism lead to the next line. Then we used $\overline{\mathrm{f}}^{\gamma} \mathrm{f}^{\beta} \otimes \overline{\mathrm{f}}_{\gamma_{1}} \overline{\mathrm{f}}^{\alpha} \mathrm{f}_{\beta} \otimes \overline{\mathrm{f}}_{\gamma_{2}} \overline{\mathrm{f}}_{\alpha}=\overline{\mathrm{f}}_{1}^{\gamma} \otimes \overline{\mathrm{f}}_{2}^{\gamma} \otimes \overline{\mathrm{f}}_{\gamma}$ which follows from the cocycle condition (2.28) by multiplying both sides of the equality with $\mathrm{f}^{\beta} \otimes \mathrm{f}_{\beta} \otimes 1$. The next step uses the antipode property $\xi_{1} S\left(\xi_{2}\right)=\varepsilon(\xi)$. Finally we used $\varepsilon\left(\overline{\mathrm{f}}^{\gamma}\right) \overline{\mathrm{f}}_{\gamma}=1$. Similarly one shows that $\chi^{-1} \chi=1$.

We are now able to prove (A.6). Starting with the left hand side we get

$$
\begin{aligned}
\mu\left(S^{\mathcal{F}} \otimes i d\right) \Delta^{\mathcal{F}}(u) & =\mu\left(S^{\mathcal{F}}\left(u_{1_{\mathcal{F}}}\right) \otimes u_{2_{\mathcal{F}}}\right) \\
& =\mathrm{f}^{\alpha} S\left(\mathrm{f}_{\alpha}\right) S\left(\mathrm{f}^{\gamma} u_{1} \overline{\mathrm{f}}^{\delta}\right) S\left(\overline{\mathrm{f}}^{\beta}\right) \overline{\mathrm{f}}_{\beta} \mathrm{f}_{\gamma} u_{2} \overline{\mathrm{f}}_{\delta} \\
& =\mathrm{f}^{\alpha} S\left(\mathrm{f}_{\alpha}\right) S\left(\overline{\mathrm{f}}^{\beta} \mathrm{f}^{\gamma} u_{1} \overline{\mathrm{f}}^{\delta}\right) \overline{\mathrm{f}}_{\beta} \mathrm{f}_{\gamma} u_{2} \overline{\mathrm{f}}_{\delta} \\
& =\mathrm{f}^{\alpha} S\left(\mathrm{f}_{\alpha}\right) S\left(u_{1} \overline{\mathrm{f}}^{\delta}\right) u_{2} \overline{\mathrm{f}}_{\delta} \\
& =\mathrm{f}^{\alpha} S\left(f_{\alpha}\right) S\left(\overline{\mathrm{f}}^{\delta}\right) S\left(u_{1}\right) u_{2} \overline{\mathrm{f}}_{\delta}
\end{aligned}
$$

Here we used that $S$ is an antialgebra homomorphism and that $\mathcal{F} \mathcal{F}^{-1}=\overline{\mathrm{f}}^{\beta} \overline{\mathrm{f}}^{\gamma} \otimes \overline{\mathrm{f}}_{\beta} \overline{\mathrm{f}}_{\gamma}=$ $1 \otimes 1$. 
Knowing that $\Delta$ is the coproduct in the $U \Xi$ Hopf algebra we find

$$
\mu(S \otimes i d) \Delta(u)=S\left(u_{1}\right) u_{2}=\varepsilon(u) .
$$

Inserting this relation into (A.7) gives

$$
\begin{aligned}
\mu\left(S^{\mathcal{F}} \otimes i d\right) \Delta^{\mathcal{F}}(u) & =\mathrm{f}^{\alpha} S\left(\mathrm{f}_{\alpha}\right) S\left(\overline{\mathrm{f}}^{\delta}\right) \varepsilon(u) \overline{\mathrm{f}}_{\delta} \\
& =\chi \chi^{-1} \varepsilon(u)=\varepsilon(u) .
\end{aligned}
$$

The right hand side of (A.6) one proves analogously.

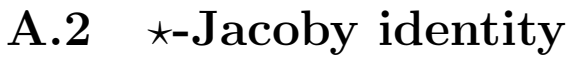

In order to prove the $\star$-Jacobi identity, $\left[u[v z]_{\star}\right]_{\star}=\left[[u v]_{\star} z\right]_{\star}+\left[\bar{R}^{\alpha}(v)\left[\bar{R}_{\alpha}(u) z\right]_{\star}\right]_{\star}$ we use the following

\section{Lemma}

$$
\overline{\mathrm{f}}^{\alpha} \bar{R}^{\gamma} \otimes \overline{\mathrm{f}}_{\alpha_{1}} \overline{\mathrm{f}}^{\beta} \bar{R}_{\gamma} \otimes \overline{\mathrm{f}}_{\alpha_{2}} \overline{\mathrm{f}}_{\beta}=\overline{\mathrm{f}}_{1}^{\alpha} \overline{\mathrm{f}}_{\delta} \otimes \overline{\mathrm{f}}_{2}^{\alpha} \overline{\mathrm{f}}^{\delta} \otimes \overline{\mathrm{f}}_{\alpha}
$$

Proof.

$$
\begin{aligned}
\overline{\mathrm{f}}^{\alpha} \bar{R} \otimes \overline{\mathrm{f}}_{\alpha_{1}} \overline{\mathrm{f}}^{\beta} \bar{R}_{\gamma} \otimes \overline{\mathrm{f}}_{\alpha_{2}} \overline{\mathrm{f}}_{\beta} & =\overline{\mathrm{f}}^{\alpha} \mathrm{f}^{\gamma} \overline{\mathrm{f}}_{\delta} \otimes \overline{\mathrm{f}}_{\alpha_{1}} \overline{\mathrm{f}}^{\beta} \mathrm{f}_{\gamma} \overline{\mathrm{f}}^{\delta} \otimes \overline{\mathrm{f}}_{\alpha_{2}} \overline{\mathrm{f}}_{\beta} \\
& =\overline{\mathrm{f}}^{\alpha} \overline{\mathrm{f}}^{\beta} \mathrm{f}^{\gamma} \overline{\mathrm{f}}_{\delta} \otimes \overline{\mathrm{f}}^{\alpha} \overline{\mathrm{f}}_{\beta} \mathrm{f}{ }_{\gamma} \overline{\mathrm{f}}^{\delta} \otimes \overline{\mathrm{f}}_{\alpha} \\
& =\overline{\mathrm{f}}_{1}^{\alpha} \overline{\mathrm{f}}_{\delta} \otimes \overline{\mathrm{f}}^{\alpha}{ }_{2} \overline{\mathrm{f}}^{\delta} \otimes \overline{\mathrm{f}}_{\alpha}
\end{aligned}
$$

where in the third line we applied property (2.28), while in the last line we used that $\overline{\mathrm{f}}^{\beta} \mathrm{f}^{\gamma} \otimes \overline{\mathrm{f}}_{\beta} \mathrm{f}_{\gamma}=\mathcal{F}^{-1} \mathcal{F}=1 \otimes 1$.

Now we observe that $\forall \xi \in U \Xi$,

$$
\mathcal{L}_{\xi}([v z])=\mathcal{L}_{\xi}(v z)-\mathcal{L}_{\xi}(z v)=\mathcal{L}_{\xi_{1}}(v) \mathcal{L}_{\xi_{2}}(z)-\mathcal{L}_{\xi_{1}}(z) \mathcal{L}_{\xi_{2}}(v)=\left[\mathcal{L}_{\xi_{1}}(v) \mathcal{L}_{\xi_{2}}(z)\right]
$$

where we used $\mathcal{L}_{\xi_{1}}(z) \mathcal{L}_{\xi_{2}}(v)=\mathcal{L}_{\xi_{2}}(z) \mathcal{L}_{\xi_{1}}(v)$ which holds because the classical coproduct $\Delta$ (see $(2.3))$ is cocommutative. Finally we have the $\star$-Jacoby identity

$$
\begin{aligned}
{\left[u[v z]_{\star}\right]_{\star} } & =\left[\overline{\mathrm{f}}^{\alpha}(u)\left[\overline{\mathrm{f}}_{\alpha_{1}} \overline{\mathrm{f}}^{\beta}(v) \overline{\mathrm{f}}_{\alpha_{2}} \overline{\mathrm{f}}_{\beta}(z)\right]\right] \\
& =\left[\overline{\mathrm{f}}_{1}^{\alpha} \overline{\mathrm{f}}^{\beta}(u)\left[\overline{\mathrm{f}}_{2}^{\alpha} \overline{\mathrm{f}}_{\beta}(v) \overline{\mathrm{f}}_{\alpha}(z)\right]\right] \\
& =\left[\left[\overline{\mathrm{f}}_{1}^{\alpha} \overline{\mathrm{f}}^{\beta}(u) \overline{\mathrm{f}}_{2}^{\alpha} \overline{\mathrm{f}}_{\beta}(v)\right] \overline{\mathrm{f}}_{\alpha}(z)\right]+\left[\overline{\mathrm{f}}_{2}^{\alpha} \overline{\mathrm{f}}_{\beta}(v)\left[\overline{\mathrm{f}}_{1}^{\alpha} \overline{\mathrm{f}}^{\beta}(u) \overline{\mathrm{f}}_{\alpha}(z)\right]\right] \\
& =\left[[u v]_{\star} z\right]_{\star}+\left[\bar{R}^{\alpha}(v)\left[\bar{R}_{\alpha}(u) z\right]_{\star}\right]_{\star}
\end{aligned}
$$

where in the second line we used property (2.28), while in the last line we used the above lemma and the fact that $U \Xi$ is cocommutative. 


\section{A.3 Associativity of the $\star-$ product on superspace}

First we calculate

$$
\begin{aligned}
(g \star h) \star k & =\mu \circ \mathcal{F}^{-1}(g \star h \otimes k) \\
& =\mu \circ \mathcal{F}^{-1}\left(\left(\mu \circ \mathcal{F}^{-1}(g \otimes h)\right) \otimes k\right) \\
& =\mu \circ \mathcal{F}^{-1} \circ\left(\left(\mu \circ \mathcal{F}^{-1}\right) \otimes i d\right)(g \otimes h \otimes k) \\
& =\mu \circ \mathcal{F}^{-1} \circ(\mu \otimes i d) \circ\left(\mathcal{F}^{-1} \otimes i d\right)(g \otimes h \otimes k) \\
& =\mu \circ(\mu \otimes i d) \circ(\Delta \otimes i d) \mathcal{F}^{-1} \circ\left(\mathcal{F}^{-1} \otimes i d\right)(g \otimes h \otimes k) \\
& =\mu \circ(\mu \otimes i d) \circ\left(\left((\Delta \otimes i d) \mathcal{F}^{-1}\right) \mathcal{F}_{12}^{-1}\right)(g \otimes h \otimes k),
\end{aligned}
$$

where in the last line we used that $\mathcal{L}_{\xi} \circ \mathcal{L}_{\zeta}=\mathcal{L}_{\xi \zeta}$ (i.e. $\xi \circ \zeta(h)=\xi \zeta(h)$ ), and in the next to last line we used that

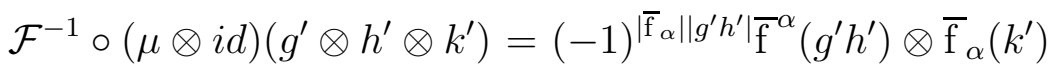

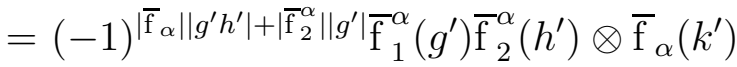

$$
\begin{aligned}
& =(\mu \otimes i d) \circ(\Delta \otimes i d) \mathcal{F}^{-1}\left(g^{\prime} \otimes h^{\prime} \otimes k^{\prime}\right) \text {. }
\end{aligned}
$$

Then we similarly obtain

$$
\begin{aligned}
g \star(h \star k) & =\mu \circ \mathcal{F}^{-1}(g \otimes(h \star k)) \\
& =\mu \circ \mathcal{F}^{-1}\left(g \otimes\left(\mu \circ \mathcal{F}^{-1}(h \otimes k)\right)\right) \\
& =\mu \circ \mathcal{F}^{-1} \circ(i d \otimes \mu) \circ\left(i d \otimes \mathcal{F}^{-1}\right)(g \otimes h \otimes k) \\
& =\mu \circ(i d \otimes \mu) \circ\left(\left((i d \otimes \Delta) \mathcal{F}^{-1}\right) \mathcal{F}_{23}^{-1}\right)(g \otimes h \otimes k)
\end{aligned}
$$

Using (2.26) we finally conclude that $(g \star h) \star k=g \star(h \star k)$, and associativity is proven.

\section{References}

[1] W. Heisenberg, Letter from Heisenberg to Peierls, in: W. Pauli, Scientific Correspondence, Vol. II, Berlin, Springer (1985).

[2] H. S. Snyder, Quantized Space-Time, Phys. Rev. 71 (1947) 38.

[3] J. Madore, Gravity on fuzzy space-time, Class. Quant. Grav. 9, 69 (1992).

[4] L. Castellani, Differential calculus on $I_{S}(N)$, quantum Poincare algebra and q-gravity, Commun. Math. Phys. 171 (1995) 383 hep-th/9312179, The Lagrangian of q-Poincare gravity, Phys. Lett. B 327 (1994) 22 hep-th/9402033. 
[5] S. Doplicher, K. Fredenhagen and J. E. Roberts, The Quantum structure of spacetime at the Planck scale and quantum fields, Commun. Math. Phys. 172 (1995) 187 hep-th/0303037, Space-time quantization induced by classical gravity, Phys. Lett. B 331 (1994) 39.

[6] A. Chamseddine, G. Felder, J. Fröhlich, Gravity in non-commutative geometry, Commun.Math.Phys. 155 (1993) 205

[7] A. Connes, Gravity coupled with matter and the foundation of non-commutative geometry, Commun. Math. Phys. 182, 155 (1996), hep-th/9603053.

[8] J. Madore and J. Mourad, Quantum space-time and classical gravity, J. Math. Phys. 39, 423 (1998), gr-qc/9607060.

[9] S. Majid, Quantum and Braided group Riemannian geometry J. Geom. Phys., 30 113-146, 1999.

[10] J. W. Moffat, Noncommutative quantum gravity, Phys. Lett. B491, 345 (2000), hep-th/0007181.

[11] A. H. Chamseddine, Deforming Einstein's gravity, Phys. Lett. B504, 33 (2001), hep-th/0009153.

[12] S. I. Vacaru, Gauge and Einstein gravity from non-Abelian gauge models on noncommutative spaces, Phys. Lett. B498, 74 (2001), hep-th/0009163.

[13] M. A. Cardella and D. Zanon, Noncommutative deformation of four dimensional Einstein gravity, Class. Quant. Grav. 20, L95 (2003), hep-th/0212071.

[14] P. Aschieri, C. Blohmann, M. Dimitrijevic, F. Meyer, P. Schupp and J. Wess, A gravity theory on noncommutative spaces, Class. Quant. Grav. 22 (2005) 3511 hepth/0504183.

[15] X. Calmet and A. Kobakhidze, Noncommutative general relativity, Phys. Rev. D 72 (2005) 045010 hep-th/0506157.

[16] G. Veneziano, A stringy nature needs just two constants Europhys. Lett. 2 (1986) 199 ;

D.J. Gross, P.F. Mende, Nucl. Phys. String theory beyond the Planck scale, B303 (1988) 407;

D.Amati, M. Ciafaloni, G. Veneziano, Superstring collisions at Planckian energy, Phys. Lett. B 197 (1987) 81; Can space-time be probed below the string size?, B 216 (1989) 41; Classical and quantum gravity effects from Planckian energy superstring collisions, Int. Jou. Mod. Phys. A 3 (1988) 1615; Higher order gravitational 
deflection and soft bremsstrahlung in Planckian energy superstring collisions, Nucl. Phys B 347 (1990) 530.

K. Konishi, G. Paffuti, P. Provero, Minimal physical length and the generalized uncertainty principle in string theory, Phys. Lett. 234 (1990) 276.

[17] T. Banks, W. Fischler, S. H. Shenker and L. Susskind, M theory as a matrix model: A conjecture, Phys. Rev. D 55 (1997) 5112, hep-th/9610043.

[18] V. G. Drinfeld, On constant quasiclassical solutions of the Yang-Baxter equations, Soviet Math. Dokl. 28 (1983) 667-671.

[19] V. G. Drinfeld, Quasi-Hopf Algebras, Lengingrad Math. J. 1 (1990) 1419 [Alg. Anal. 1N6 (1989) 114].

[20] N. Reshetikhin, Multiparameter Quantum Groups And Twisted Quasitriangular Hopf Algebras, Lett. Math. Phys. 20 (1990) 331.

[21] C. Jambor and A. Sykora, Realization of algebras with the help of *-products, hep-th/0405268.

[22] M. Gerstenhaber, A. Giaquinto, S. D. Schack, in Quantum symmetry. Proceedings in EIMI 1990, Lect. Notes Math. 1510 ed. P. P. Kulish (Springer-Verlag, Berlin, 1992) pp. 9-46.

[23] O. V. Ogievetsky, in Proc. Winter School Geometry and Physics, Zidkov, Suppl. Rendiconti cir. Math. Palermo, Serie II - N 37, 185 (1993); preprint MPI-Ph/92-99, Munich, (1992) 14p.

[24] P.P. Kulish, V.D. Lyakhovsky, A.I. Mudrov Extended jordanian twists for Lie algebras, J. Math. Phys. 40, 4569 (1999), math.QA/9806014,

P.P. Kulish, V.D. Lyakhovsky and M.A. del Olmo, Chains of twists for classical Lie algebras, J. Phys. A: Math. Gen. 32, 8671 (1999), math.QA/9908061.

[25] P. Aschieri and L. Castellani, Bicovariant Calculus on Twisted ISO(N), Quantum Poincare' Group and Quantum Minkowski Space, Int. J. Mod. Phys. A 11 (1996) 4513 q-alg/9601006, $R$ matrix formulation of the quantum inhomogeneous group $I S O-q, r(N)$ and ISp-q,r(N), Lett. Math. Phys. 36 (1996) 197 hep-th/9411039.

[26] P.P. Kulish, A.I. Mudrov Twist-related geometries on q-Minkowski space Proc. Steklov Inst. Math. 226 (1999) 97-111, math.QA/9901019.

[27] J. Wess, Deformed coordinate spaces: Derivatives, Lecture given at the Balkan workshop BW2003, August 2003, Vrnjacka Banja, Serbia, hep-th/0408080. 
[28] M. Chaichian, P. Kulish, K. Nishijima, and A. Tureanu, On a Lorentz-invariant interpretation of noncommutative space-time and its implications on noncommutative QFT, Phys. Lett. B604, 98 (2004), hep-th/0408069.

M. Chaichian, P. Presnajder and A. Tureanu, New concept of relativistic invariance in NC space-time: Twisted Poincare symmetry and its implications, Phys. Rev. Lett. 94 (2005) 151602 hep-th/0409096.

[29] F. Koch and E. Tsouchnika, Construction of $\theta$-Poincare algebras and their invariants on $M_{\theta}$, Nucl. Phys. B 717 (2005) 387 hep-th/0409012.

[30] C. Gonera, P. Kosinski, P. Maslanka and S. Giller, Space-time symmetry of noncommutative field theory, Phys. Lett. B 622 (2005) 192 hep-th/0504132, Global symmetries of noncommutative space-time, Phys. Rev. D 72 (2005) 067702 hepth/0507054.

[31] R. Oeckl, Untwisting noncommutative $R^{d}$ and the equivalence of quantum field theories, Nucl. Phys. B581, 559 (2000), hep-th/0003018.

[32] J. Lukierski and M. Woronowicz, New Lie-algebraic and quadratic deformations of Minkowski space from twisted Poincare symmetries, hep-th/0508083.

[33] A. Connes and G. Landi, Noncommutative manifolds: The instanton algebra and isospectral deformations, Commun. Math. Phys. 221, 141 (2001) math.qa/0011194.

[34] A. Connes and M. Dubois-Violette, Noncommutative finite-dimensional manifolds. I. Spherical manifolds and related examples, math.qa/0107070.

[35] V. Gayral, B. Iochum and J. C. Varilly, Dixmier traces on noncompact isospectral deformations, hep-th/0507206.

[36] J. C. Varilly, Quantum symmetry groups of noncommutative spheres, Commun. Math. Phys. 221 (2001) 511 math.qa/0102065.

[37] M. Rieffel, Deformation quantization for actions of $\mathbb{R}^{d}$, Memoris AMS, vol. 506, AMS, Providence (1993).

[38] A. Sitarz, Twists and spectral triples for isospectral deformations, Lett. Math. Phys. 58 (2001) 69-79, math.QA/0102074, Rieffel's deformation quantization and isospectral deformations. Int. J. Theor. Phys. 40 (2001) 1693. math.QA/0102075.

[39] P. Aschieri and F. Bonechi, On the Noncommutative Geometry of Twisted Spheres, Lett. Math. Phys. 59 (2002) 133 math.qa/0108136.

[40] S. L. Woronowicz, Differential Calculus On Compact Matrix Pseudogroups (Quantum Groups), Commun. Math. Phys. 122 (1989) 125. 
[41] P. Aschieri and L. Castellani, An Introduction to noncommutative differential geometry on quantum groups, Int. J. Mod. Phys. A 8 (1993) 1667 hep-th/9207084.

[42] P. Schupp, P. Watts and B. Zumino, Bicovariant quantum algebras and quantum Lie algebras, Commun. Math. Phys. 157 (1993) 305 hep-th/9210150.

[43] P. Aschieri, On the geometry of inhomogeneous quantum groups, math.qa/9805119, Scuola Normale Superiore di Pisa, Pubblicazioni Classe di Scienze, Collana Tesi.

[44] P. Aschieri et al. In preparation.

[45] M. Dimitrijević and J. Wess, Deformed bialgebra of diffeomorphisms, Lecture given by M. D. at the 1st Vienna Central European Seminar, November 26th-28th, 2004, Vienna, Austria, hep-th/0411224.

[46] F. Meyer, Noncommutative spaces and Gravity, Lecture given at the first Modave Summer School in Mathematical Physics, June 2005, Modave (Belgium), hep-th/0510188

[47] M.E. Sweedler, Hopf Algebras, Benjamin, New Yourk (1969), E. Abe, Hopf Algebras, Cambridge University Press, Cambridge (1980).

[48] S. Majid, Foundations of quantum group theory, Cambridge: University Press (1995) $606 \mathrm{p}$.

[49] V. Chari and A. Pressley, A Guide to Quantum Groups, Cambridge: University Press (1995) $651 \mathrm{p}$.

[50] C. Brouder, B. Fauser, A. Frabetti and R. Oeckl, Quantum field theory and Hopf algebra cohomology, J. Phys. A 37 (2004) 5895 hep-th/0311253.

[51] S. Ferrara and M. A. Lledo, JHEP 0005, 008 (2000) hep-th/0002084, D. Klemm, S. Penati and L. Tamassia, Non(anti)commutative superspace, Class. Quant. Grav. 20 (2003) 2905 hep-th/0104190.

J. de Boer, P. A. Grassi and P. van Nieuwenhuizen, Non-commutative superspace from string theory, Phys. Lett. B 574, 98 (2003) hep-th/0302078.

N. Seiberg, Noncommutative superspace, $N=1 / 2$ supersymmetry, field theory and string theory, JHEP 0306 (2003) 010 hep-th/0305248.

[52] Y. Kobayashi and S. Sasaki, Lorentz invariant and supersymmetric interpretation of noncommutative quantum field theory, hep-th/0410164.

B. M. Zupnik, Twist-deformed supersymmetries in non-anticommutative superspaces, Phys. Lett. B 627 (2005) 208 hep-th/0506043.

M. Ihl and C. Sämann, Drinfeld-twisted supersymmetry and non-anticommutative superspace hep-th/0506057. 
[53] D. Gurevich and S. Majid, Braided groups of Hopf algebras obtained by twisting, Pac. J. Math. 162:27-44, 1994.

[54] S. Majid and R. Oeckl, Twisting of quantum differentials and the Planck scale Hopf algebra, Commun. Math. Phys. 205 (1999) 617 math.qa/9811054.

[55] B. L. Cerchiai, G. Fiore and J. Madore, Geometrical Tools for Quantum Euclidean Spaces, Commun. Math. Phys. 217 (2001) 521 math.qa/0002007.

[56] Joseph C. Varilly, Hector Figueroa, Jose M. Gracia-Bondia Elements of Noncommutative Geometry Birkhäuser Advanced Texts / Basler Lehrbacher (2000).

[57] L. Conlon, Differentiable Manifolds Birkhäuser Advanced Texts / Basler Lehrbacher (1st ed. 1994 or 2nd ed. 2001). 